\title{
Enthalpic and Entropic Contributions to Interleaflet Coupling Drive Domain Registration and Anti-registration in Biological Membrane
}

\author{
Akshara Sharma* \\ Department of Physics, Indian Institute of Science-Bangalore, \\ C. V. Raman Road, Bangalore, Karnataka 560012, India \\ Aniruddha Seal \\ School of Chemical Sciences, \\ National Institute of Science Education and Research, \\ Bhubaneswar, Khurda, Odisha 752050, India \\ Sahithya S. Iyer $I^{\dagger}$ and Anand Srivastave \\ Molecular Biophysics Unit, Indian Institute of Science-Bangalore, \\ C. V. Raman Road, Bangalore, Karnataka 560012, India
}

(Dated: September 29, 2021)

\begin{abstract}
Biological membrane is a complex self-assembly of lipids, sterols and proteins organized as a fluid bilayer of two closely stacked lipid leaflets. Differential molecular interactions among its diverse constituents give rise to heterogeneities in the membrane lateral organization. Under certain conditions, heterogeneities in the two leaflets can be spatially synchronised and exist as registered domains across the bilayer. Several contrasting theories behind mechanisms that induce registration of nanoscale domains have been suggested [1] [3]. Following a recent study [4] showing the effect of position of lipid tail unsaturation on domain registration behavior, we decided to develop an analytical theory to elucidate the driving forces that create and maintain domain registry across leaflets. Towards this, we formulated a Hamiltonian for a stacked lattice system where site variables encapsulate the lipid molecular properties including the position of unsaturation and various other interactions that could drive phase separation and interleaflet coupling. We solve the Hamiltonian using Monte Carlo simulations and create a complete phase diagram that reports the presence or absence of registered domains as a function of various Hamiltonian parameters. We find that the interleaflet coupling should be described as a competing enthalpic contribution due to interaction of lipid tail termini, primarily due to saturated-saturated interactions, and an interleaflet entropic contribution from overlap of unsaturated tail termini. We find that higher position of unsaturation provides weaker interleaflet coupling. We also find points in our parameter space that allow thermodynamically stable nanodomains in our bilayer model, which we have verified by carrying out extended Monte Carlo simulations. These persistent non-coalescing registered nanodomains close to the lower end of the accepted nanodomain size range also point towards a possible "nanoscale" emulsion description of lateral heterogeneities in biological membrane leaflets.
\end{abstract}

\section{INTRODUCTION}

The biological cell membrane contains a host of lipids and other biomolecules such as proteins and glycans, which interact dynamically to facilitate biological processes like signal transduction and membrane protein oligomerization. Lipids in model ternary and quaternary membrane systems can segregate into liquid ordered $\left(L_{o}\right)$ and liquid disordered $\left(L_{d}\right)$ phases. Experimentally observed phase separated domains from in vitro studies range from micrometers to nanometers in their size [512. Apart from studies on model in vitro systems, segregation of lipids into $L_{o}$ and $L_{d}$ phases has also been

\footnotetext{
* Also at Molecular Biophysics Unit, Indian Institute of ScienceBangalore.

$\dagger$ Currently at Department of Chemistry, University of Illinois Chicago, 845 W. Taylor St., 4500 SES (MC 111), Chicago, IL 60607, U.S.A

‡ anand@iisc.ac.in
}

observed in in vivo membranes [13 16]. The functional importance of this phase separation is exemplified during signal transduction across cells, where the relay of signal from the outer to inner leaflet and vice-versa is highly dependent on the communication between the two leaflets. Experimental studies have shown the importance of lipids in synchronising the reception and transmission of messages across the bilayer 17-19]. T-cell and B-cell receptor mediated immune response is a classical example of systems where co-localization of outer and inner leaflet ordered domains, i.e "registration" of ordered lipids, plays an important role in lipid mediated signal transduction [20-22]. Understanding the origin and molecular driving forces giving rise to "registration" or "anti-registration" of the ordered domains in the two leaflets can provide important insights into the related physiologically critical processes.

Existing studies on the topic contain a variety of theories, some of them conflicting with each other, on the nature of the driving forces for domain registration. Exchange of cholesterol between ordered domains in the 
outer leaflet and the inner leaflet in asymmetric membranes has been hypothesised to form and maintain registered ordered domains in the inner leaflet in some theoretical models 23 25]. A mismatch energy, contributed when ordered and disordered domains overlap between leaflets, has also been studied as a possible driving force for domain registration [1. This mismatch energy can be understood in terms of the energy penalty required when $L_{o}$ and $L_{d}$ domains interact between leaflets. In contrast, however, several studies claim membrane undulations and domain bending stiffness to cause domain registration without any direct interleaflet coupling [3, 26]. There is also work done suggesting domain boundary line tension as a driving force for registration and this model also does not require explicit coupling of the lipid bilayer leaflets 27. These studies argue that the difference in splay rigidities between the $L_{o}$ and $L_{d}$ domains in both leaflets causes preferential distribution of stiffer domains in regions of the membrane with lower curvature fluctuations. This, along with the energy gain from a decrease of line tension at the domain boundaries, is suggested as a possible explanation towards a driving force for registration of both large and small domains. There have been theoretical and computational studies on another candidate for a possible driving force, hydrophobic mismatch, arising due to a difference in tail lengths leading to differential membrane thickness between $L_{o}$ and $L_{d}$ domains [2, 16, 28, 31]. Both domain formation kinetics and registration dynamics have been studied using hydrophobic mismatch as a driving force, and it has been theoretically shown to produce phase coexistence metastable states that capture both registered and antiregistered possibilities upon phase separation.

While there is agreement that there isn't one single driving force for this phenomenon, the complexity of the problem comes from the number of hypotheses available that seem to explain domain registration or antiregistration. Further additions have been made to this list of candidates recently, of particular interest being a computational study by S. Zhang and X. Lin 4] showing a significant effect of the position of unsaturation along the lipid tail on domain registration tendencies in otherwise identical systems. The authors carried out MARTINI 32 coarse-grained molecular dynamics (CGMD) simulations of two systems that were a mixture of DPPC, Cholesterol and an unsaturated lipid with different positions of unsaturation, which they called D23 and D34. Fig 1 shows the chemical structures of the lipids.

They observed that the system where the unsaturated lipid had lower position of unsaturation showed registration and the other system showed anti-registration. They also suggested that the interleaflet coupling was through an attractive interaction in the interleaflet region between the tail termini of the lipids.

We found this to be a very intriguing face of the problem, and decided to investigate the physical cause of the position of unsaturation affecting domain registration characteristics, which we believed could provide im- portant insights into the overall registration mechanism. Besides testing the deductions made in the original work that credited the enthalpic interaction in the interleaflet region for driving registration, we also wanted to explore the role of the competing entropic factors in the interleaflet region. Our primary hypothesis is as follows: A higher position of unsaturation would lead to reduced configurational entropy of the lipid tails in the core of the membrane leading to a reduction in interleaflet interaction. On the other hand, a lower position of unsaturation forces the tail terminus to explore the interleaflet region (instead of bending towards the polar headgroups), which would lead to not only a better enthalpic but also an enhanced entropic contribution to the interleaflet coupling. The entropic aspect of interleaflet coupling is an important and often overlooked aspect that we bring to light in this work.

To test this, we formulate a Hamiltonian with tunable parameters that captures the hypothesised enthalpic and entropic contributions as a function of position of unsaturation along the length of the lipid tail for a lattice system representing the bilayer as a stack of two square lattices. We used this Hamiltonian and conducted a parameter study using Monte Carlo simulations for each point in parameter space for all the systems under investigation. Using these simulations, we try to capture the most dominant factor affecting the registration characteristics of the systems, as well as the effect of the change in position of unsaturation for a system described by our Hamiltonian. The schematic of our workflow is provided in sec.I of the Supporting Information (S.I) as fig:S1.

We describe our lattice model of the membrane bilayer in the "Model" section below and provide simulation details, specifications of the studied systems, as well as details about our analysis tools in the "Methods" section. Following that, we report our observations and findings in the "Results" section and we put all our results in the context of existing literature and provide a detailed perspective on the implications of our observations in the "Discussion" section. We finally summarize our work

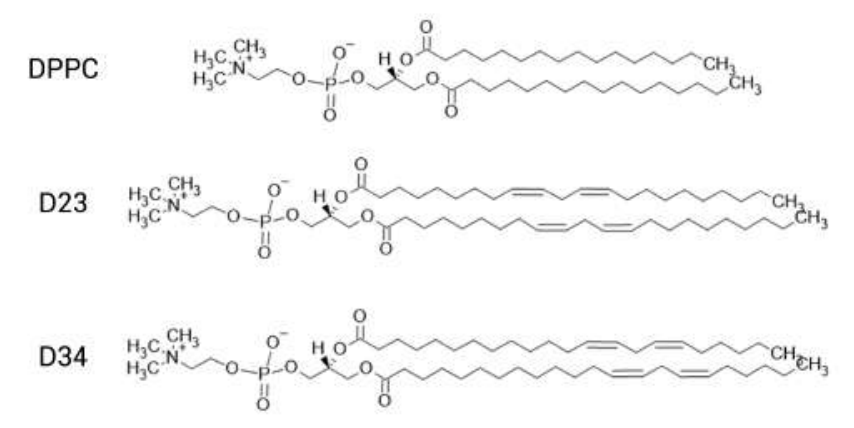

FIG. 1: The chemical structures of the DPPC, D23 and D34 lipids. Note that the D23 and D34 lipids differ only in their position of unsaturation and do not occur naturally. 
in the "Conclusions" section with some perspectives on position of unsaturation in lipids as a driving force for domain registration.

\section{MODEL}

Following our hypothesis, we wrote a Hamiltonian for a system with two coupled membrane leaflets that captured entropic behaviour of the lipid tails as well as the positions of unsaturations in the lipid tails. For this, we decided to model the system as a pair of stacked square lattices, each square representing a membrane leaflet. Each leaflet would be populated with lipids in a symmetric population distribution. Our site variables for the lattice Hamiltonian are carefully curated to capture the molecular details of the lipid, which we describe below. Our Hamiltonian can be broken into two independent parts, one for the lateral interactions within a leaflet, and the other for the interleaflet interactions.

$$
H=H_{\text {lat }}+H_{\text {inter }}
$$

Both $H_{\text {lat }}$ and $H_{\text {inter }}$ consist of an enthalpic and an entropic part. $H_{\text {lateral }}$ is formulated as shown below where the first term can be denoted as $H_{\text {lat }}^{\text {enth }}$ and the second term as $H_{\text {lat }}^{\text {entr }}$.

$$
\begin{aligned}
H_{l a t}= & \frac{1}{2} \sum_{<i, j>} \sum_{k=0,1}\left(\sum_{\alpha, \alpha^{\prime}} \epsilon_{\alpha \alpha^{\prime}} \delta_{\alpha, i k} \delta_{\alpha^{\prime}, j k}\right) \\
& +\frac{V_{S} k_{B} T}{4} \sum_{k=0,1} \sum_{i}\left(n_{\alpha, i k} \ln \left(\phi_{\alpha, i k}\right)\right. \\
& \left.+n_{\alpha^{\prime}, i k} \ln \left(\phi_{\alpha^{\prime}, i k}\right)\right)
\end{aligned}
$$

Similarily, $H_{\text {inter }}$ is formulated as shown below, where the first term can be denoted as $H_{\text {inter }}^{\text {enth }}$ and the second term as $H_{\text {inter }}^{\text {entr }}$.

$$
\begin{aligned}
H_{\text {inter }}= & \sum_{i}\left(\sum_{\alpha, \alpha^{\prime}} \epsilon_{\alpha \alpha^{\prime}}^{\prime} \delta_{\alpha, i 0} \delta_{\alpha^{\prime}, i 1}\right) \\
& +V_{S}^{\prime} k_{B} T \sum_{k=0,1} \sum_{i}\left(\left(1+\frac{\sum p_{\alpha}}{l_{\alpha}}\right) n_{\alpha, i k}^{\prime} \ln \left(\phi_{\alpha, i k}^{\prime}\right)\right. \\
& \left.+\left(1+\frac{\sum p_{\alpha^{\prime}}}{l_{\alpha^{\prime}}}\right) n_{\alpha^{\prime}, i k}^{\prime} \ln \left(\phi_{\alpha^{\prime}, i k}^{\prime}\right)\right)
\end{aligned}
$$

In the above eqns 2 and 3 the summation $\langle i, j\rangle$ is a sum over orthogonal neighbours within a leaflet, and the sum over $\mathrm{i}$ is a sum over the sites of a leaflet. The $\delta_{\alpha, i k}$ are kronecker deltas that become 1 when the lipid occupying site $\mathrm{i}$ in leaflet $\mathrm{k}$ is of species $\alpha$. We used a simple Ising-like enthalpic interaction between lipids in a leaflet for the lateral part at site $\mathrm{i}$ as follows:

$$
\begin{gathered}
\epsilon_{\alpha \alpha^{\prime}}=-V_{\alpha \alpha^{\prime}} s_{\alpha, i 0} s_{\alpha^{\prime}, j 0} \\
H_{l a t}^{\text {enth }}=\frac{1}{2} \sum_{<i, j>} \sum_{k=0,1}\left(\sum_{\alpha, \alpha^{\prime}} \epsilon_{\alpha \alpha^{\prime}} \delta_{\alpha, i k} \delta_{\alpha^{\prime}, j k}\right)
\end{gathered}
$$

and for lipids opposite each other in the two leaflets for the interleaflet part as shown:

$$
\begin{gathered}
\epsilon_{\alpha \alpha^{\prime}}^{\prime}=-V_{\alpha \alpha^{\prime}}^{\prime} s_{\alpha, i 0} s_{\alpha^{\prime}, i 1} \\
H_{\text {inter }}^{\text {enth }}=\sum_{i} \sum_{\alpha, \alpha^{\prime}} \epsilon_{\alpha \alpha^{\prime}}^{\prime} \delta_{\alpha, i 0} \delta_{\alpha^{\prime}, i 1}
\end{gathered}
$$

where $V_{\alpha \alpha^{\prime}}$ and $V_{\alpha \alpha^{\prime}}^{\prime}$ are the corresponding interaction strength parameters that can be tuned and serve as a design variable. The site variables $s_{\alpha, i k}$ for species $\alpha$ at site $\mathrm{i}$ in leaflet $k=0$ or 1 is given by:

$$
s_{\alpha, i k}=\sum_{c=1}^{l_{\alpha}} \frac{S_{\alpha, c}}{l_{\alpha}}
$$

which is the average $S_{C D}$ of the lipid species occupying the site $\mathrm{i}$ in leaflet $\mathrm{k}$, calculated from lipid trajectories generated from all-atom molecular dynamics (AAMD) simulations described ahead. As can be seen, the forms in eqns 4 and 6 would lead to a stronger interleaflet enthalpic contribution for a lower position of unsaturation due to the higher average $S_{C D}$ of those lipids.

We define the following term for the entropic behaviour in the plane of a leaflet, where $n_{\alpha, i k}$ is the count of lipids of species $\alpha$ in the local region of site i in leaflet $\mathrm{k}$ shown in fig 2(a) and $\phi_{\alpha, i k}$ is the fraction of lipids of species $\alpha$ in the same local region.

$$
H_{l a t, i}^{e n t r}=\frac{V_{S} k_{B} T}{4}\left(n_{\alpha, i k} \ln \left(\phi_{\alpha, i k}\right)+n_{\alpha^{\prime}, i k} \ln \left(\phi_{\alpha^{\prime}, i k}\right)\right)
$$

This, summed over all sites of both leaflets, the above equation can be expressed as:

$$
\begin{aligned}
H_{\text {lat }}^{\text {entr }}=\frac{V_{S} k_{B} T}{4} \sum_{k=0,1} \sum_{i} & \left(n_{\alpha, i k} \ln \left(\phi_{\alpha, i k}\right)\right. \\
+ & \left.n_{\alpha^{\prime}, i k} \ln \left(\phi_{\alpha^{\prime}, i k}\right)\right)
\end{aligned}
$$

For the entropic interaction coupling the two leaflets, we define

$$
\begin{aligned}
H_{\text {inter }, i}^{\text {entr }}=V_{S}^{\prime} k_{B} T & \left(\left(1+\frac{\sum p_{\alpha}}{l_{\alpha}}\right) n_{\alpha, i k}^{\prime} \ln \left(\phi_{\alpha, i k}^{\prime}\right)\right. \\
& \left.+\left(1+\frac{\sum p_{\alpha^{\prime}}}{l_{\alpha^{\prime}}}\right) n_{\alpha^{\prime}, i k}^{\prime} \ln \left(\phi_{\alpha^{\prime}, i k}^{\prime}\right)\right)
\end{aligned}
$$


(a)

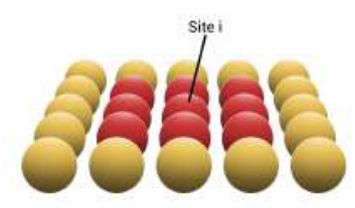

(b)

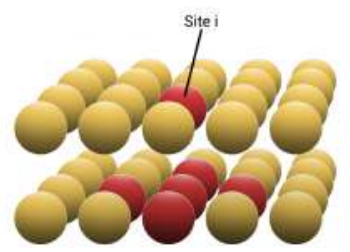

FIG. 2: Cartoon representation of a local region of the model, where the red sites are the locality over which the parameters $n_{\alpha}$ and $\phi_{\alpha}$ are calculated for a lattice site i. (a) represents the sites for the entropic term of $H_{\text {lateral }}$, and (b) represents the sites for the entropic term of $H_{\text {interleaflet }}$

which, when summed over all sites of both leaflets, becomes

$$
\begin{aligned}
H_{\text {inter }}^{\text {entr }}=V_{S}^{\prime} k_{B} T \sum_{k=0,1} \sum_{i} & \left(\left(1+\frac{\sum p_{\alpha}}{l_{\alpha}}\right) n_{\alpha, i k}^{\prime} \ln \left(\phi_{\alpha, i k}^{\prime}\right)\right. \\
+ & \left.\left(1+\frac{\sum p_{\alpha^{\prime}}}{l_{\alpha^{\prime}}}\right) n_{\alpha^{\prime}, i k}^{\prime} \ln \left(\phi_{\alpha^{\prime}, i k}^{\prime}\right)\right)
\end{aligned}
$$

where $p_{\alpha} / l_{\alpha}$ gives the fractional position of unsaturation along the lipid tail, being 0 at the head group, and 1 at the end of the tail, which is then summed over all unsaturations of the tail. This scaling of the entropic terms ensures that the entropic contribution is stronger for terms involving lipids with lower position of unsaturation while the saturated lipids remain unaffected. $n_{\alpha}^{\prime}$ and $\phi_{\alpha}^{\prime}$ are calculated in a locality shown in fig $2(\mathrm{~b})$.

For a system of two lipids $\mathrm{A}$ and $\mathrm{B}$, this gives us a Hamiltonian with 9 parameters that are tunable during simulations, shown together for convenience in table Ibelow.

We use the Hamiltonian as defined above to calculate the energy for our systems in the Monte-Carlo simulations used in this study, which we describe in the following section.

\section{METHODS}

We begin this section by describing how we obtained the site variables and describing the systems we studied, followed by how they were used to generate data using Monte-Carlo simulations. We also provide details of the analysis tools used to obtain quantitative results on phase separation and domain registration tendencies of the systems from the generated data.
TABLE I: Description of all the tunable independent parameters of the Hamiltonian for a 2-component

\begin{tabular}{|c|c|}
\hline Parameter & Description \\
\hline $\mathrm{T}$ & Temperature \\
\hline$V_{S}$ & Lateral entropic term strength constant \\
\hline$V_{S}^{\prime}$ & Interleaflet entropic term strength constant \\
\hline$V_{A A}$ & $\begin{array}{l}\text { Lateral enthalpic strength constant for A-A } \\
\text { interaction }\end{array}$ \\
\hline$V_{B B}$ & $\begin{array}{l}\text { Lateral enthalpic strength constant for B-B } \\
\text { interaction }\end{array}$ \\
\hline$V_{A B}$ & $\begin{array}{l}\text { Lateral enthalpic strength constant for A-B } \\
\text { interaction }\end{array}$ \\
\hline$V_{A A}^{\prime}$ & $\begin{array}{l}\text { Interleaflet enthalpic strength constant for } \\
\text { A-A interaction }\end{array}$ \\
\hline$V_{B B}^{\prime}$ & $\begin{array}{l}\text { Interleaflet enthalpic strength constant for B- } \\
\mathrm{B} \text { interaction }\end{array}$ \\
\hline$V_{A B}^{\prime}$ & $\begin{array}{l}\text { Interleaflet enthalpic strength constant for } \\
\text { A-B interaction }\end{array}$ \\
\hline
\end{tabular}
system with lipid species A and B.

\section{A. All-atom simulations of artificial lipids}

We train the values of site variable (see eqn 8) for each of the lipids used in the Monte-Carlo simulations from all atom trajectories of DPPC-D23-Chol and DPPC-D34-Chol systems. The study by S. Zhang and X. Lin 4 used CGMD simulations where D23 and D34 are artificial lipids. To capture the lipid molecular properties faithfully, we reconstructed an atomic resolution system and carried out AAMD simulations. In our work, we have used a prescription that allows us to build all-atom structures for artificial lipids that have a similar chemical makeup to lipids with well-known forcefield parameters. We provide a schematic of the workflow in fig 3 and we describe the steps in the following text. We prepared the all-atom descriptions of the artificial lipids, namely D23 and D34 lipids using the Ligand Reader and Modeler Module in CHARMM-GUI33, 34. Since we aren't using partial charges from Quantum-Chemical calculations, we need to check the assigned partial charges with a closely matching parameterized lipid molecule which, in this case, were the partial charges on the $\mathrm{sp}^{2}$ and $\mathrm{sp}^{3}$ hybridised lipid tail carbons in the lipid DUPC. We then build the multi-component lipid bilayer using the MemGen webserver 35 based on the percentage composition of the required bilayer and the number of lipids per leaflet in our system. We have organized a tutorial with examples for the workflow described above and made it publicly available at https://github.com/codesrivastavalab/ Membrane-Nanodomain-Registration/tree/main/ Artificial\%20Lipid. Also, please note that common modelling errors and issues during the generation of the all-atom bilayer systems might require some troubleshooting, which we discuss in sec.2 of the S.I.

For our systems, the average $S_{C D}$ was calculated for 


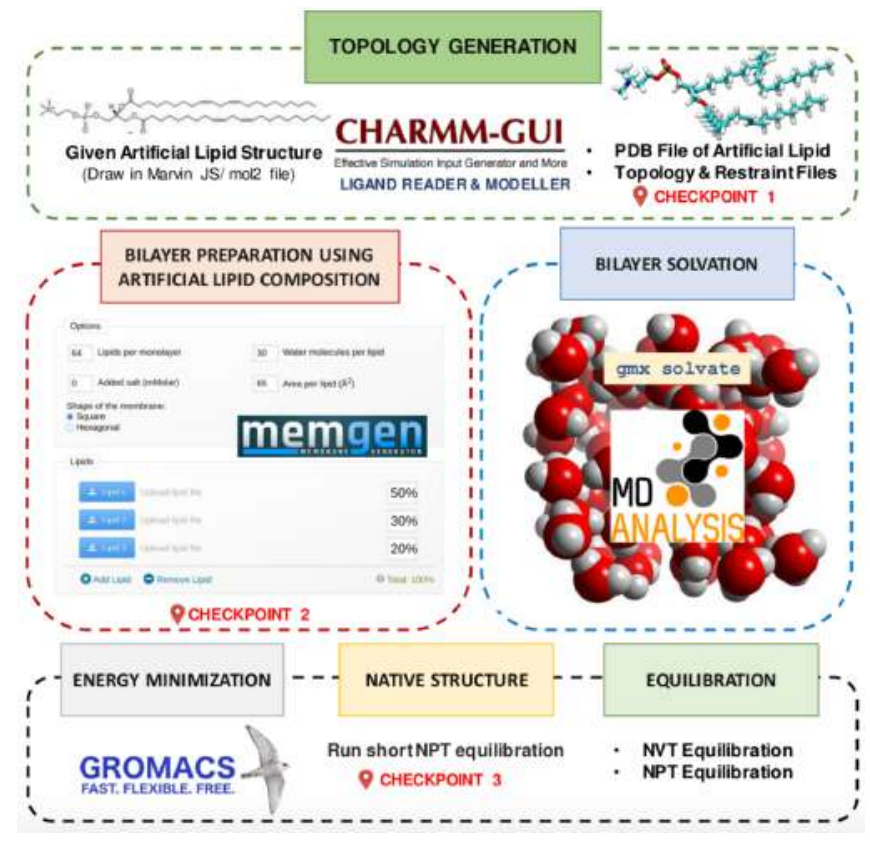

FIG. 3: Schematic of the workflow for generating artificial lipid force field parameters for lipids with different position of unsaturation and generation of the bilayer topology.

DPPC, D23 and D34 lipids from the all-atom system of 144 DPPC, 80 D23/D34, and 56 Chol molecules in each leaflet, which is close to the ratio used by Zhang and Lin [4] in their publication. We used the CHARMM36 force field and first minimized the energy of the system using steepest descent minimization. Then, we carried out two rounds of 125 ps NVT equilibration at 303.15 $\mathrm{K}$ and four rounds of $250 \mathrm{ps}$ NPT equilibration. We ran production runs using GROMACS 36, 37, for a period of $1 \mu s$ with the PME[38, 39] method for electrostatics, Nose-Hoover [40] temperature coupling and ParinelloRahman 41 pressure coupling. The LINCS 42 algorithm was used to restrain hydrogen bonds. The tail order parameters of D23 and D34 lipids were calculated using the gmx order program in GROMACS. The $S_{C D}$ values obtained are averaged over all the D23/D34 lipids in the D23/D34, DPPC, Chol ternary mixture and across 7.5 ns ( $S_{C D}$ remain the same with increased averaging time). $S_{C D}$ of $n^{\text {th }}$ carbon is calculated using the position of $n-1$ and $n+1$ carbons.

Fig 4 shows the $S_{C D}$ values for D23 and D34 lipids. The decrease in the $S_{C D}$ values occurs at the position of double bonds in D23 and D34 lipids. The dip in value of $S_{C D}$ for the C atoms near the end of the alkyl tails of D34 lipids indicates the lower position of the double bonds as seen from the molecular structure of the lipids. On an average, D34 lipids show more ordering of the alkyl tails in comparison to D23 lipids. The final values of the site variables calculated by averaging the $S_{C D}$ over all tail carbon atoms for the DPPC-D23 systems were DPPC0.3074681, D23-0.1692048, and for the DPPC-D34 sys- (a)

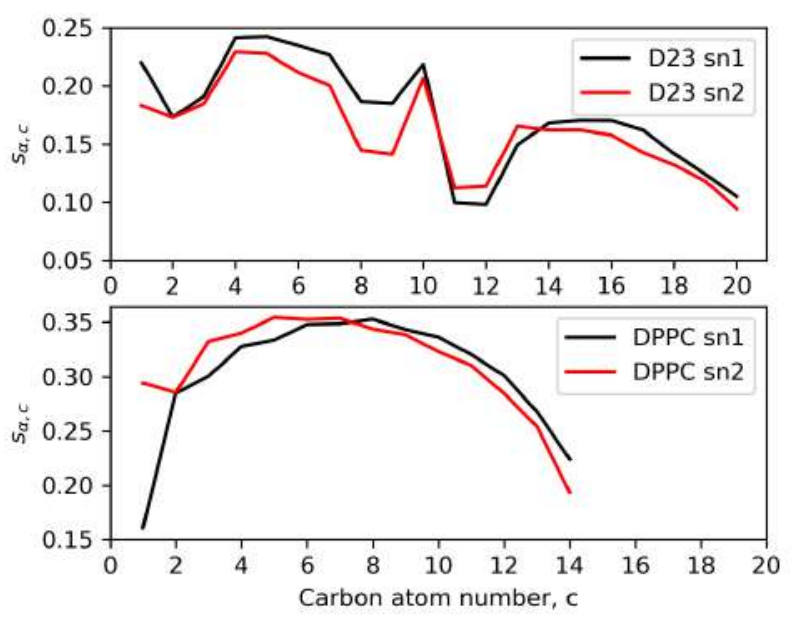

(b)

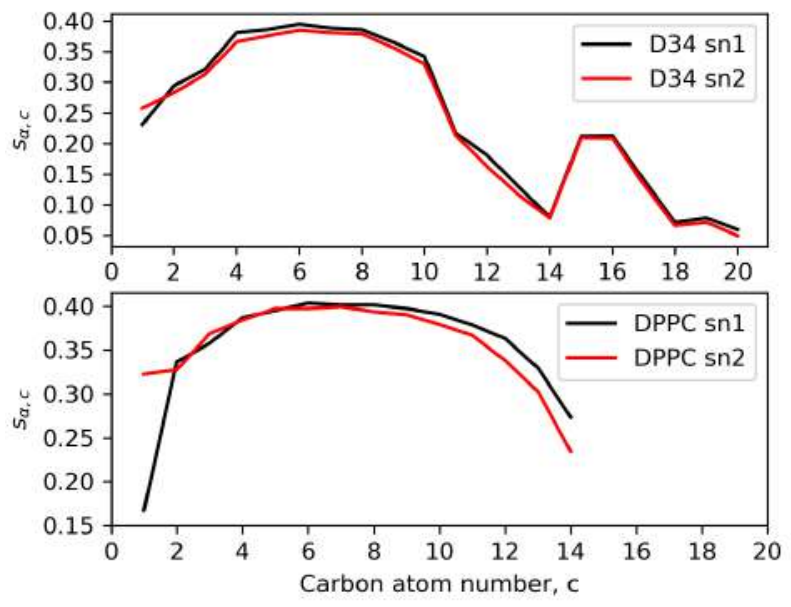

FIG. 4: a) shows the $S_{C D}$ for the tail carbon atoms of the D23 and DPPC lipids as calculated by averaging over all D23 lipids of the D23-DPPC system. b) Shows the same for the D34-DPPC system.

tems, they were DPPC-0.3565843, D34-0.2400412. As we can see, the average $S_{C D}$ is higher for the unsaturated lipid with a lower position of unsaturation. Please note that the purpose of the AAMD simulations is only to extract out a more realistic site variable for our Hamiltonian.

\section{B. Setup for Monte Carlo simulations}

We studied 6 systems, each being a stack of $2100 \times$ 100 square lattices. The upper and lower leaflet have symmetric populations in each system, with no lattice points left empty. So, each leaflet contains 10,000 lipids 
of different species depending on the system. The details of the 6 systems are shown in table III.

TABLE II: Composition of the bilayers of the 6 systems with symmetric leaflets.

\begin{tabular}{lc}
\hline \hline Components & System Composition \\
\hline DPPC, D23 & $2 \times(5000$ DPPC, 5000 D23 $)$ \\
DPPC, D23 & $2 \times(2500$ DPPC, 7500 D23 $)$ \\
DPPC, D23 & $2 \times(7500$ DPPC, 2500 D23 $)$ \\
DPPC, D34 & $2 \times(5000$ DPPC, 5000 D34 $)$ \\
DPPC, D34 & $2 \times(2500$ DPPC, 7500 D34 $)$ \\
DPPC, D34 & $2 \times(7500$ DPPC, 2500 D34 $)$ \\
\hline \hline
\end{tabular}

Each system underwent a Monte-Carlo simulation with traditional Metropolis acceptance [43] for each combination of parameters in the following parameter space in reduced units

$$
\begin{aligned}
& T=2.048 \\
& V_{S}=0.06 \\
& V_{S}^{\prime} \in\{0.005,0.02,0.035,0.050\} \\
& V_{A A}^{\prime} \in\{8.0,20.0,32.0\} \\
& V_{B B}^{\prime} \in\{8.0,20.0,32.0\} \\
& V_{A B}^{\prime} \in\{8.0,20.0,32.0\} \\
& V_{A A} \in\{4.0,7.0,10.0,13.0,16.0\} \\
& V_{B B} \in\{4.0,7.0,10.0,13.0,16.0\} \\
& V_{A B} \in\{4.0,7.0,10.0,13.0,16.0\}
\end{aligned}
$$

with the restriction that $V_{A B} \leq V_{B B} \leq V_{A A}$, since points outside this would not show phase separation. This gives us 3780 points in parameter space for each of the 6 systems. The temperature in reduced units corresponds to the temperature of the all-atom simulations from which the site variables were calculated. Also, we chose the range of the in-plane enthalpic strength constants such that the interaction energy would lie in the neighbourhood of the physiological DPPC-DPPC interaction energy estimated by P. Almeida in 2009 44]. Further references to any point in parameter space will be written as a chain of indices of parameters in the order shown in eqn 13 above. For example, point 0-0-0-2-1-0-4-4-0 refers to the parameters $T=2.048, V_{S}=0.06, V_{S}^{\prime}=0.005$, $V_{A A}^{\prime}=32.0, V_{B B}^{\prime}=20.0, V_{A B}^{\prime}=8.0, V_{A A}^{\prime}=16.0$, $V_{B B}=16.0, V_{A B}=4.0$.

The systems for each point in parameter space were initialized by randomly choosing from their two components at each lattice site, with a check to ensure we didn't exceed the specified population for the chosen species. This caused the initial configurations to have regions of one or the other species, but this was lost very quickly in a few MC moves during the simulations, thereby not affecting the converged state of each system for any point in the parameter space. Each initialized system corresponding to a point in the parameter space underwent Monte-Carlo simulations for $10^{7}$ moves, each move consisting of an exchange attempt of site variables between two randomly chosen sites, first for the upper leaflet followed by an attempt for the lower leaflet separately. The system configurations were output every 5000 moves, giving us 2000 configurations for each point in parameter space for each system. The last 200 configurations were used for analysis. The Monte-Carlo data for each point in parameter space for each of the 6 systems was further analysed using tools as described ahead.

\section{Analysis tools}

Owing to the large number of parameter space points for each of the 6 systems, it is impractical to visually classify the phase separaton and domain registration of each point in parameter space, so we wrote simple $\mathrm{C}$ routines to do the classification for us without visual aid, whose functioning is described below. The programs used for the analysis described here as well as that for the MC simulations described in the previous section have been made publicly available at https://github.com/codesrivastavalab/ Membrane-Nanodomain-Registration/tree/main/ Lipid\%20Domain\%20Registration

\section{Quantification of phase separation using domain size distributions}

Nanoscale molecular-level heterogeneity and structures are non-trivial to quantify [4-48]. We used a Depth-First Search (DFS) based algorithm to determine domain size distributions for each leaflet for a given configuration to categorize them as phase-separated(PS), not phaseseparated(NPS) or partially phase separated(PPS) based on a consistent set of cutoffs. If $>80 \%$ of lipids of each species were in domains of size $>1600$ lipids, they were categorized as PS, if $>80 \%$ of lipids of either species were in domains of size $<400$ lipids, they were considered NPS. Any configurations not in either category were considered to be PPS. A point in parameter space was assigned one of the three states if a majority of configurations from the last $10 \%$ of output configurations in it's Monte-Carlo simulation were of that state. Although the cut-offs are somewhat arbitrary, since they are applied consistently to all of the systems, the results still show reliable qualitative trends with the variation of the tunable parameters.

\section{Domain registration quantification using KL Divergence}

For domain registration, we used KullbackLeibler(KL) divergence 49] of the two leaflets as a measure of registration. KL Divergence (denoted by $D_{K L}$ ) was the tool of choice for us because of its simplicity as well as its ability to clearly and quantitatively separate systems based on fixed cut-offs. $D_{K L}$ for a pair 
of probability distributions $\mathrm{P}(\mathrm{x})$ and $\mathrm{Q}(\mathrm{x})$ on a random variable $\mathrm{x}$ on the same probability space is given by

$$
D_{K L}(P \| Q)=\sum_{x \in \chi} P(x) \log \left(\frac{P(x)}{Q(x)}\right)
$$

where $\mathrm{P}(\mathrm{x})$ and $\mathrm{Q}(\mathrm{x})$ are constructed from a $2 \mathrm{D}$ leaflet configuration of the upper and lower leaflet respectively from the Monte-Carlo trajectories of a system with lipids $\mathrm{A}$ and $\mathrm{B}$ as follows

$$
P(i, j)=\left\{\begin{array}{l}
\frac{0.5}{0.5 N_{A}+1.0 N_{B}}, \text { if A occupies site }(\mathrm{i}, \mathrm{j}), \\
\frac{1.0}{0.5 N_{A}+1.0 N_{B}}, \text { if B occupies site }(\mathrm{i}, \mathrm{j})
\end{array}\right.
$$

where $N_{A}$ and $N_{B}$ are the populations of lipids of species $\mathrm{A}$ and $\mathrm{B}$, respectively.

$\mathrm{Q}(\mathrm{i}, \mathrm{j})$ is identically defined, but in the lower leaflet. The choice of weights 0.5 and 1 is arbitrary and it doesn't affect our categorization as we rescale the $D_{K L}$ values of each system to lie between 0 and 1 before continuing with classification of the data. Configurations were categorized as registered (R), partially registered (PR), unregistered (UR), partially anti-registered (PAR), and anti-registered (AR) based on cut-off values of $D_{K L}$ at $0.1,0.3,0.7$, and 0.9 , which correspond to $10 \%, 30 \%, 70 \%$, and $90 \%$ of the lipids of lowest population in mismatched configurations between leaflets. Once again, the category of the majority of the analysed configurations from the Monte Carlo simulations of a point in parameter space was assigned to that point.

\section{RESULTS}

In this section, we describe the results of the simulations and analysis that we discussed above. We first look at the results of the MC simulations. We have reported the convergence of the simulations in section III of the SI, where we have shown the energy profiles of randomly selected points from across the parameter space to show sufficient convergence of the MC simulations. The Hamiltonian that we wrote can successfully capture phase separation in leaflets, and can capture different extents of domain registration and anti-registration behaviour. Fig 5 shows some select configurations generated at various points in parameter space in our systems.

\section{A. Interleaflet entropic contributions suppress phase separation}

After confirming that all of the simulations had converged, we used the classifying method for phase separation described earlier on the last 200 configurations (last $10 \%$ ) of the MC data for each point in parameter space for each system. This classified each point into phase separated (PS), partially phase separated (PPS) or not a)
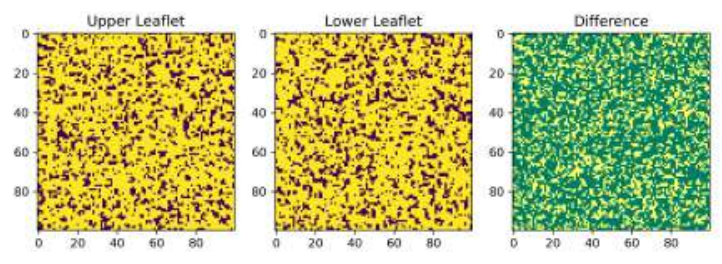

b)
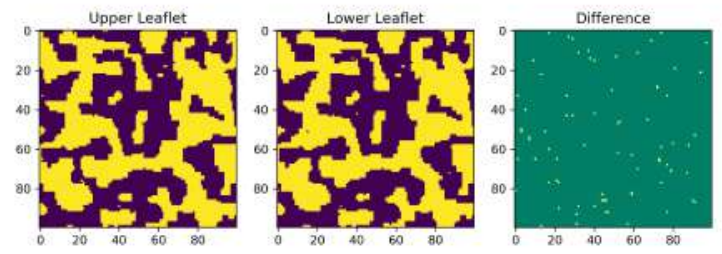

c)
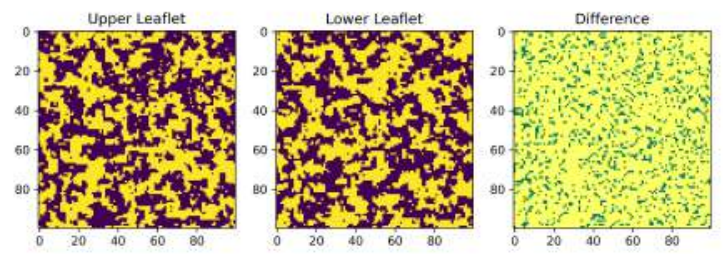

d)
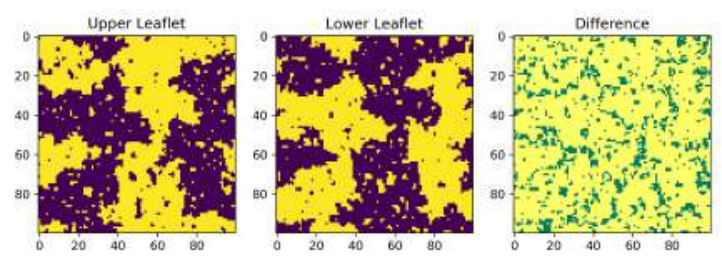

FIG. 5: In the shown leaflet configurations, yellow represents DX sites and purple represents DPPC sites. In the difference plot, yellow represents a mismatch and green represents a match between leaflets at that site.

a) shows the upper and lower leaflet configurations with their difference for parameters $0-0-0-1-1-1-2-2-0$ for the

1:3 DPPC-D34 system, which is not phase separated

(NPS, UR), b) shows the same for parameters $0-0-0-2-0-0-4-4-0$ for the 1:1 DPPC-D34 system which is phase separated and registered (PS, R), c) for parameters 0-0-0-2-0-2-4-0-0 for the 1:1 DPPC-D34 system which is partially phase separated and partially anti-registered (PPS, PAR), and d) for parameters 0-0-1-2-2-2-4-4-0 for the 1:1 DPPC-D34 system which is phase separated and anti-registered (PS, AR).

phase separated (NPS), which we then plotted as a parameter space plot with the variation in the Hamiltonian parameters. However, due to 7 varying parameters out of the 9 tunable parameters, seeing each variation required the use of a complicated nested plot of plots, which we describe here. The plots shown in fig 6, fig:7, and fig 8 below as well as fig:S3, fig:S4, and fig:S5 in sec.IV of the S.I each have an outer pair of axes, the horizontal axis representing $V_{B B}^{\prime}$ and the vertical one representing $V_{S}^{\prime}$. Nested within this pair of axes are 12 subplots and each of the 12 subplots have a pair of axes such that the horizontal one represents $V_{A B}^{\prime}$ and the vertical one represents $V_{A A}^{\prime}$. Within each of these subplots, there are $93 \mathrm{D}$ plots which have three axes representing the parameters $V_{A A}$, 
bioRxiv preprint doi: https://doi.org/10.1101/2021.09.29.462263; this version posted September 30, 2021. The copyright holder for this preprint (which was not certified by peer review) is the author/funder, who has granted bioRxiv a license to display the preprint in perpetuity. It is made available under aCC-BY 4.0 International license.

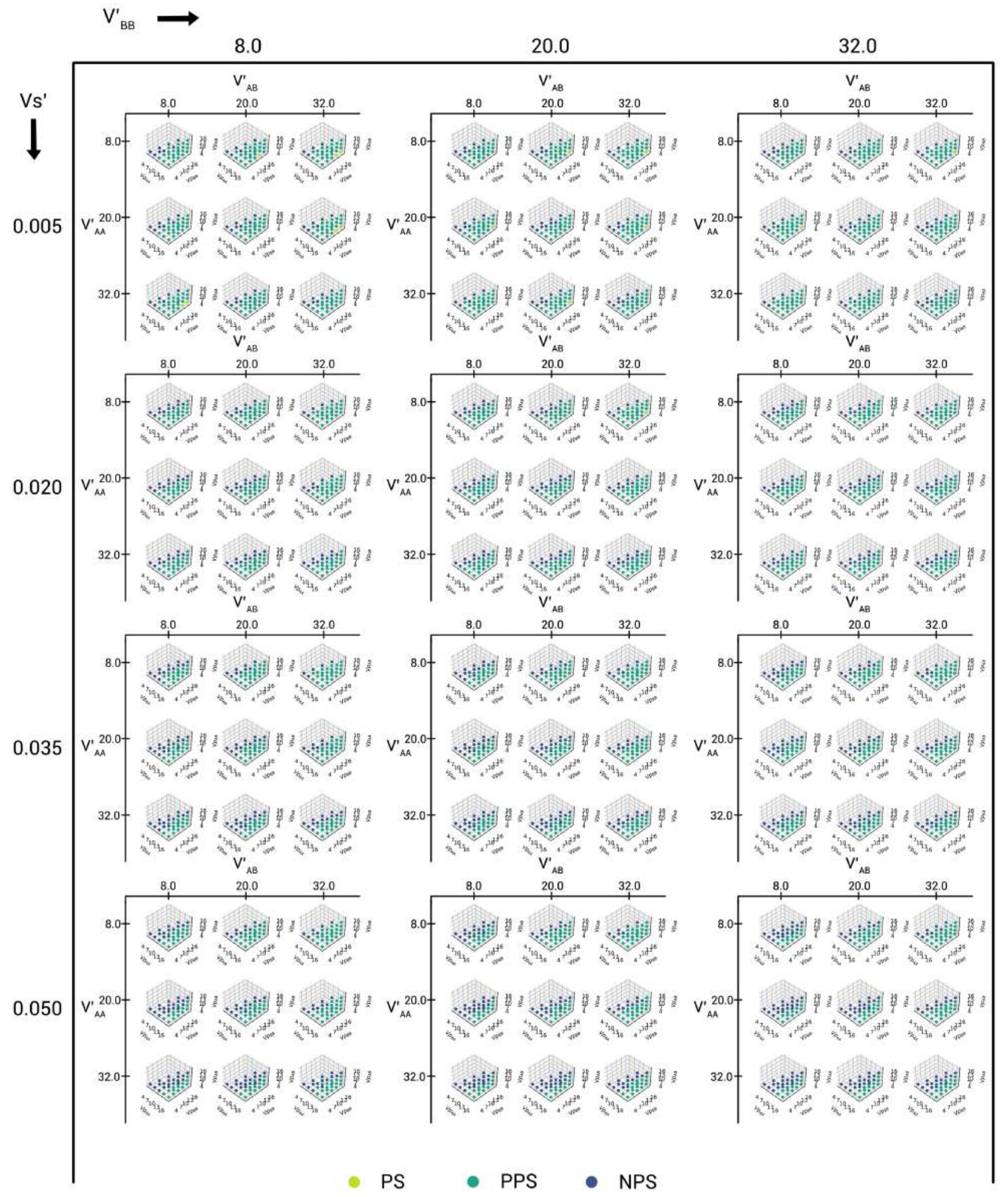

FIG. 6: The plot of phase separation classification for all of the parameter space points for the 1:1 DPPC-D23 system, assigned using the domain size distribution analysis. 
bioRxiv preprint doi: https://doi.org/10.1101/2021.09.29.462263; this version posted September 30, 2021. The copyright holder for this preprint (which was not certified by peer review) is the author/funder, who has granted bioRxiv a license to display the preprint in perpetuity. It is made available under aCC-BY 4.0 International license.

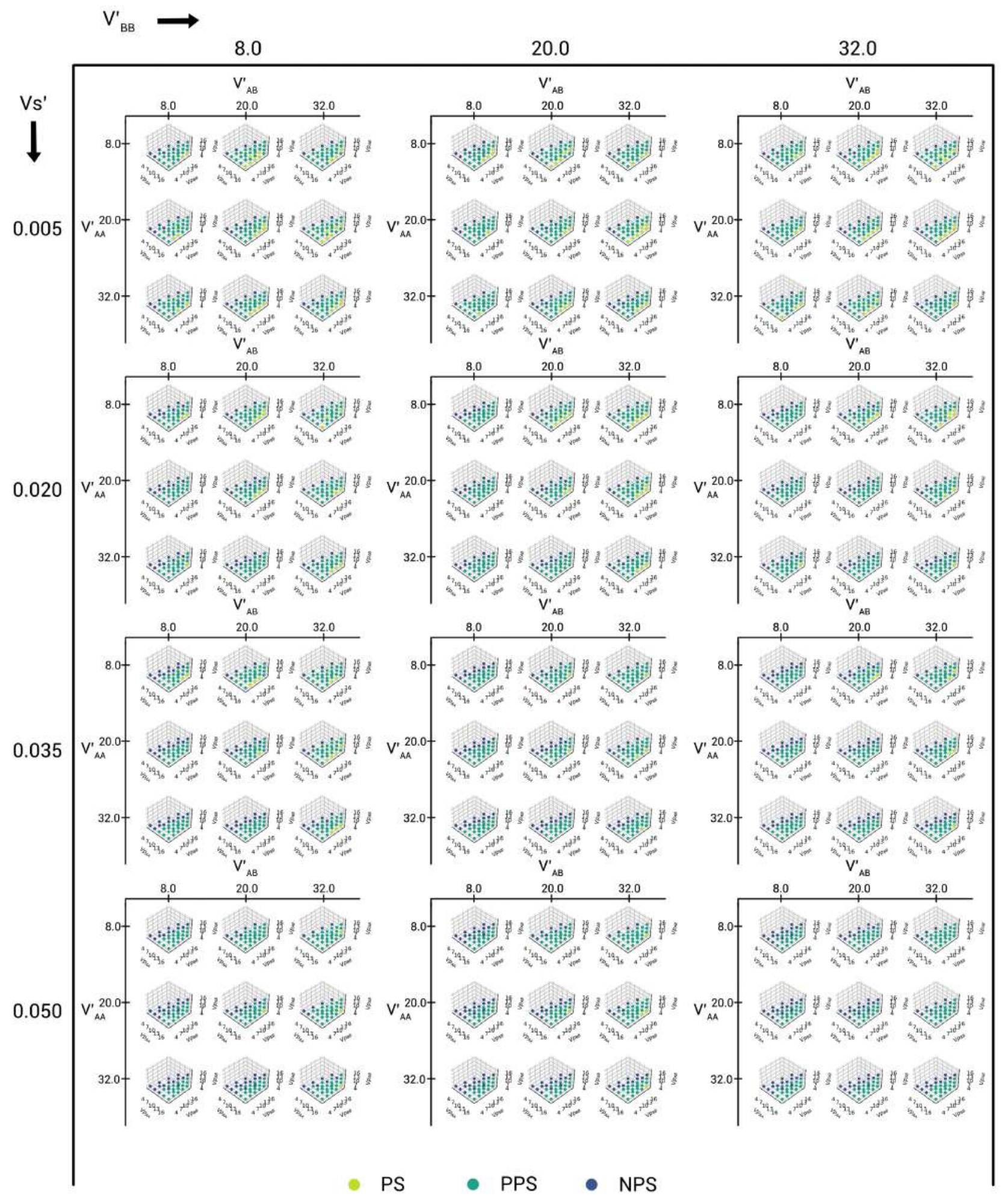

FIG. 7: The plot of phase separation classification for all of the parameter space points for the 1:1 DPPC-D34 system, assigned using the domain size distribution analysis. 
bioRxiv preprint doi: https://doi.org/10.1101/2021.09.29.462263; this version posted September 30, 2021. The copyright holder for this preprint (which was not certified by peer review) is the author/funder, who has granted bioRxiv a license to display the preprint in perpetuity. It is made available under aCC-BY 4.0 International license.

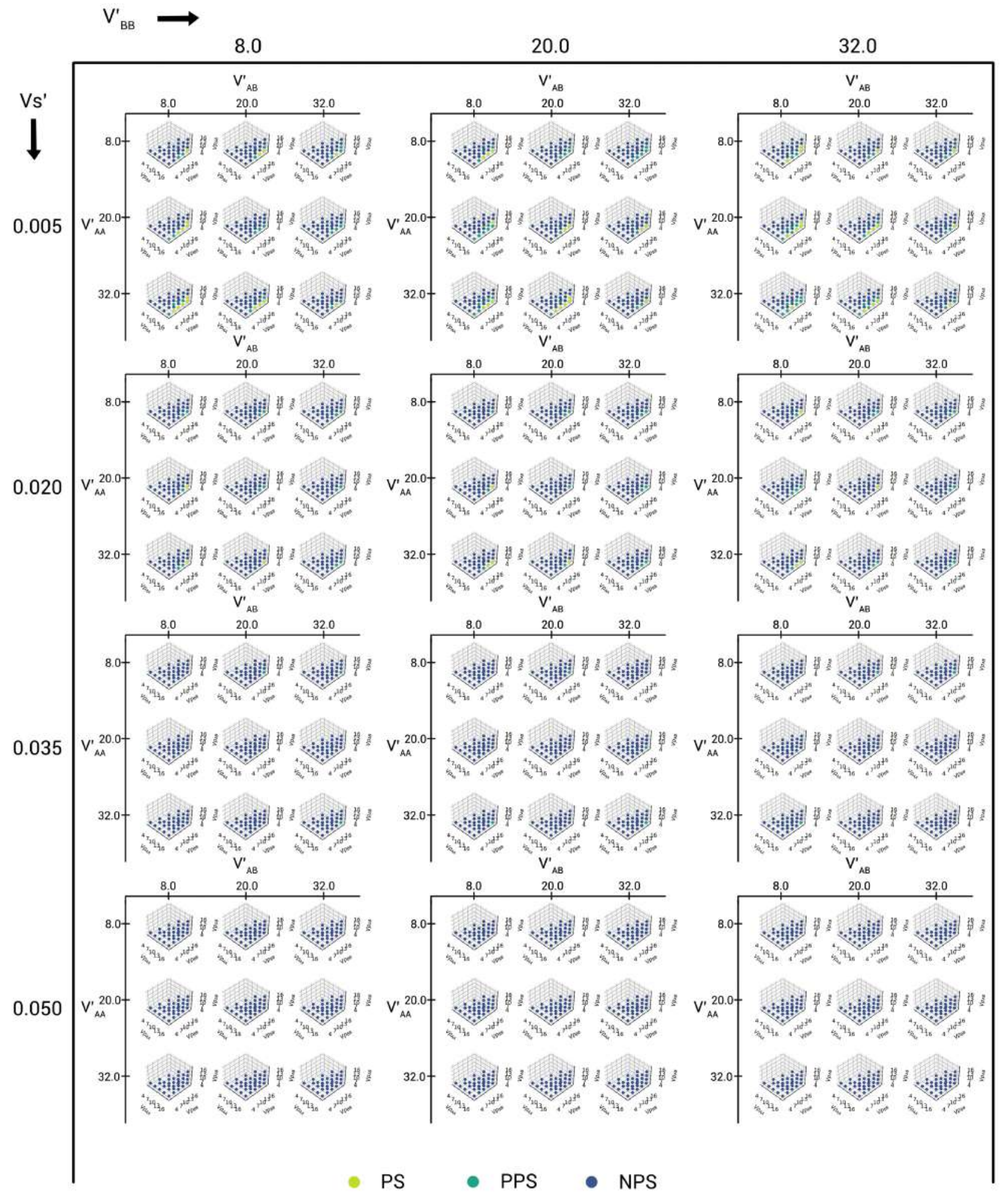

FIG. 8: The plot of phase separation classification for all of the parameter space points for the 1:3 DPPC-D34 system, assigned using the domain size distribution analysis. 
$V_{B B}$, and $V_{A B}$. This effectively shows the variation of classification state with variation in the 7 changing parameters in a $2 \mathrm{D}$ plot. The first and most intuitive observation can be made from the increase in propensity to phase separate with increasing $V_{A A}$ and $V_{B B}$ values and a decrease in propensity to phase separate with increase in $V_{A B}$ values. This effect on phase separation, being purely enthalpic in its origin, is reflected in all the 6 systems studied, which is an unsurprising but reassuring observation to establish the fidelity of our modeling work. In another observation, we find that increase in $V_{A A}^{\prime}, V_{B B}^{\prime}$ and $V_{A B}^{\prime}$ values in the systems independently results in a slightly increased propensity to phase separate. Since these interaction parameters control inter-leaflet interactions, their effect on phase separation reflects their role in domain registration/anti-registration. Another general trend that we see is that $V_{S}^{\prime}$ reduces the number of phase separated points as it increases. This suppression of lateral phase separation due to the interleaflet entropic interaction is a surprising result that emerges from our chosen form for the Hamiltonian.

Looking specifically at fig 6 and fig 7 we can see the qualitative differences between the D23 and D34 systems. The overall extent of phase separation is higher in the 1:1 DPPC-D34 system, which indicates expected behaviour. We can also see from fig 8 that there are very few phaseseparated points in parameter space. This is also the case with the 1:3 DPPC-D23 system and the two 3:1 systems whose plots are shown in fig:S3, fig:S4, and fig:S5 in sec.IV] of the S.I. This shows that a skew away from a 1:1 population ratio can also severely hinder phaseseparation. This could be understood as an emergent effect of the lateral and interleaflet entropic terms in our Hamiltonian. However, the 1:3 systems show slightly better phase separation than the $3: 1$ systems, which clearly shows the effect of our interleaflet entropic interaction term, as shown in eqn 12, where we rescale the contributions to represent the effect of position of unsaturation. We therefore find that the systems with higher populations of unsaturated lipids phase separate better than those with higher populations of saturated lipids in each leaflet. We will now proceed to look at the parameter space plots for domain registration before drawing any conclusions about the behaviour of the systems.

\section{B. Anti-registration is promoted by interleaflet entropy}

Similar to the previous analysis, we used the last $10 \%$ of the configurations from the Monte carlo simulations of each point in parameter space to conduct the analysis and categorization of domain registration into 5 categories, $\mathrm{R}$, $\mathrm{PR}, \mathrm{UR}, \mathrm{PAR}$, and AR. Once again, we made parameter space plots of this classification for the 6 systems that we studied, which are shown in fig 9, fig 10, and fig 11 below as well as fig:S6, fig:S7, and fig:S8 in sec.V of the S.I. These plots have the same structure as described earlier, and can therefore be read in the same manner.

Again, we first look at the general trends in the plots for our 6 systems. First, we notice that the parameters $V_{A A}, V_{B B}$, and $V_{A B}$ do not have any direct correlation with the extent of domain registration. This would mean that these parameters only directly influence the phase separation of the systems within the leaflets. We also see that the parameters $V_{A A}^{\prime}$ and $V_{B B}^{\prime}$ promote domain registration, whereas the parameter $V_{A B}^{\prime}$ promotes domain anti-registration. This too, is an intuitive result. The interesting trend that we find here is that of $V_{S}^{\prime}$. Upon increasing, it promotes domain anti-registration and suppresses domain registration. Also, going back to the parameters $V_{A A}^{\prime}, V_{B B}^{\prime}$, and $V_{A B}^{\prime}$ from the phase separation plots, we see that the phase-separated regions there correspond to either registered or anti-registered parts of the plots here. So, what we actually see is that $V_{A A}^{\prime}$ and $V_{B B}^{\prime}$ promote phase separation when domain registration is favoured, and $V_{A B}^{\prime}$ favours phase separation when domain anti-registration is favoured.

By comparing the DPPC-D23 systems and DPPCD34 systems, we find that the DPPC-D34 systems have a larger fraction of points in the $\mathrm{R}$ or $\mathrm{AR}$ categories, whereas the DPPC-D23 systems have most of their points in the PR, UR and PAR categories. This can be seen clearly in fig 9, fig [10 and fig.11] below as well as the fig:S6, fig:S7, and fig:S8 in sec.V of the S.I. This would suggest that the energy landscape of the DPPCD34 system in parameter space has a sharper transition from favouring registration to favouring anti-registration, whereas the DPPC-D23 system has a smoother energy landscape in parameter space. We also find that skewing the ratio of DPPC-D23 or DPPC-D34 away from 1:1 leads to a suppression of domain registration and a significant increase in anti-registration in both $1: 3$ and $3: 1$ ratio systems. Also, the parameter space plots for the 1:3 and 3:1 systems for both D23 and D34 lipids are almost identical, suggesting that the increase in anti-registration in this case has to do mostly with the population ratio and not the specific lipids involved. The extent of the change, however, depends on the lipids involved, as we see a more significant dominance of anti-registration in the DPPC-D34 systems with the 1:3 and 3:1 population ratios in their leaflets. There is, however, a slightly higher number of registered points in the 1:3 systems over the $3: 1$ systems, as can be seen upon carefully inspecting the plot for the 1:3 DPPC-D34 system given in fig 11] and the 3:1 DPPC-D34 system shown in fig:S8 in the S.I.

\section{Thermodynamically stable nanodomains}

We also found some intriguing results while observing the converged states of some of the points in the parameter space for our systems with skewed population. There were some converged states that showed configurations consisting almost entirely of separate domains that did not grow very large in size, but did not scatter 
bioRxiv preprint doi: https://doi.org/10.1101/2021.09.29.462263; this version posted September 30, 2021. The copyright holder for this preprint (which was not certified by peer review) is the author/funder, who has granted bioRxiv a license to display the preprint in perpetuity. It is made available under aCC-BY 4.0 International license.

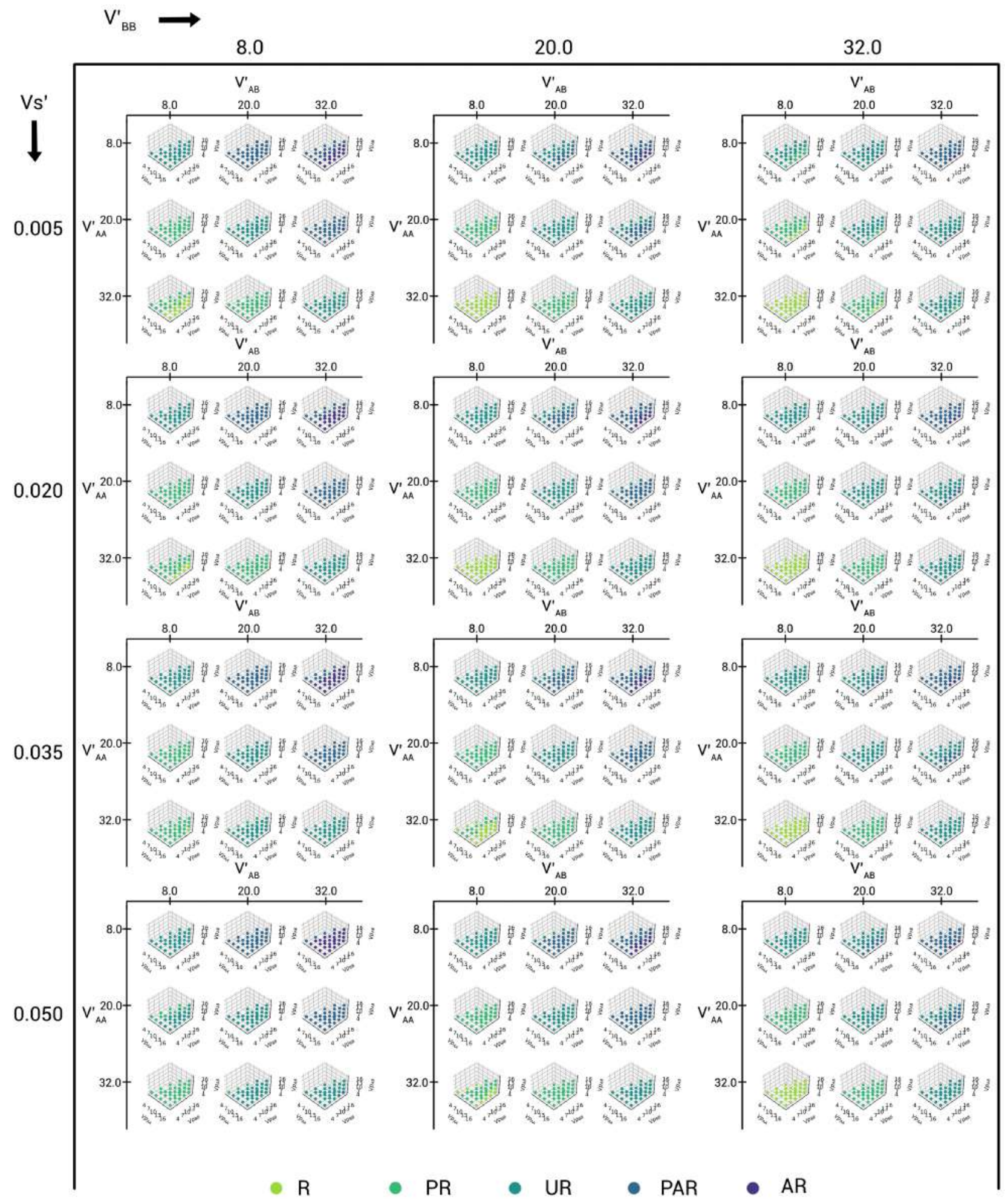

FIG. 9: The plot of domain registration behaviour for all of the parameter space points for the 1:1 DPPC-D23 system, assigned using the KL Divergence analysis. 
bioRxiv preprint doi: https://doi.org/10.1101/2021.09.29.462263; this version posted September 30, 2021. The copyright holder for this preprint (which was not certified by peer review) is the author/funder, who has granted bioRxiv a license to display the preprint in perpetuity. It is made available under aCC-BY 4.0 International license.

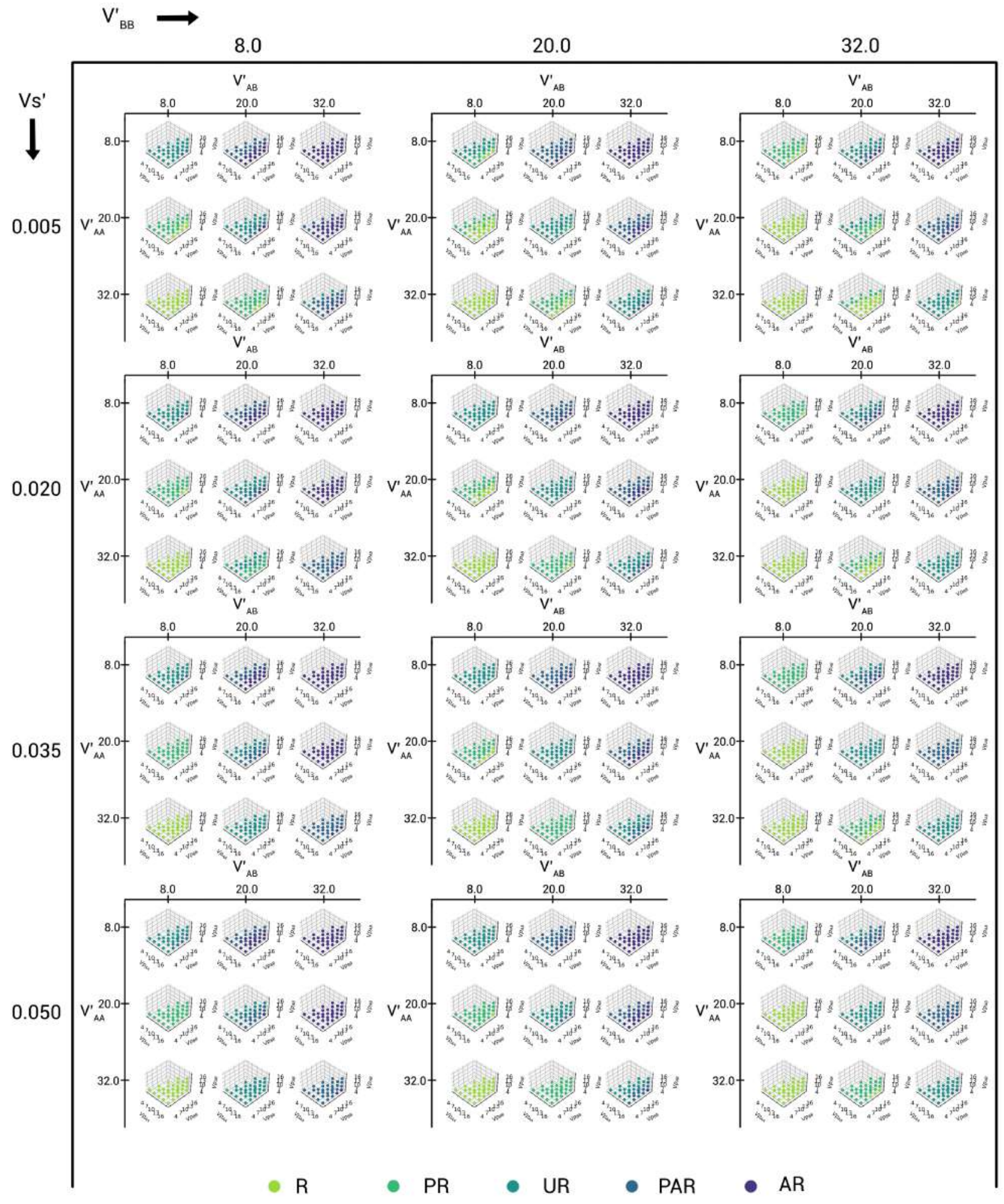

FIG. 10: The plot of domain registration behaviour for all of the parameter space points for the 1:1 DPPC-D34 system, assigned using the KL Divergence analysis. 
bioRxiv preprint doi: https://doi.org/10.1101/2021.09.29.462263; this version posted September 30, 2021. The copyright holder for this preprint (which was not certified by peer review) is the author/funder, who has granted bioRxiv a license to display the preprint in perpetuity. It is made available under aCC-BY 4.0 International license.

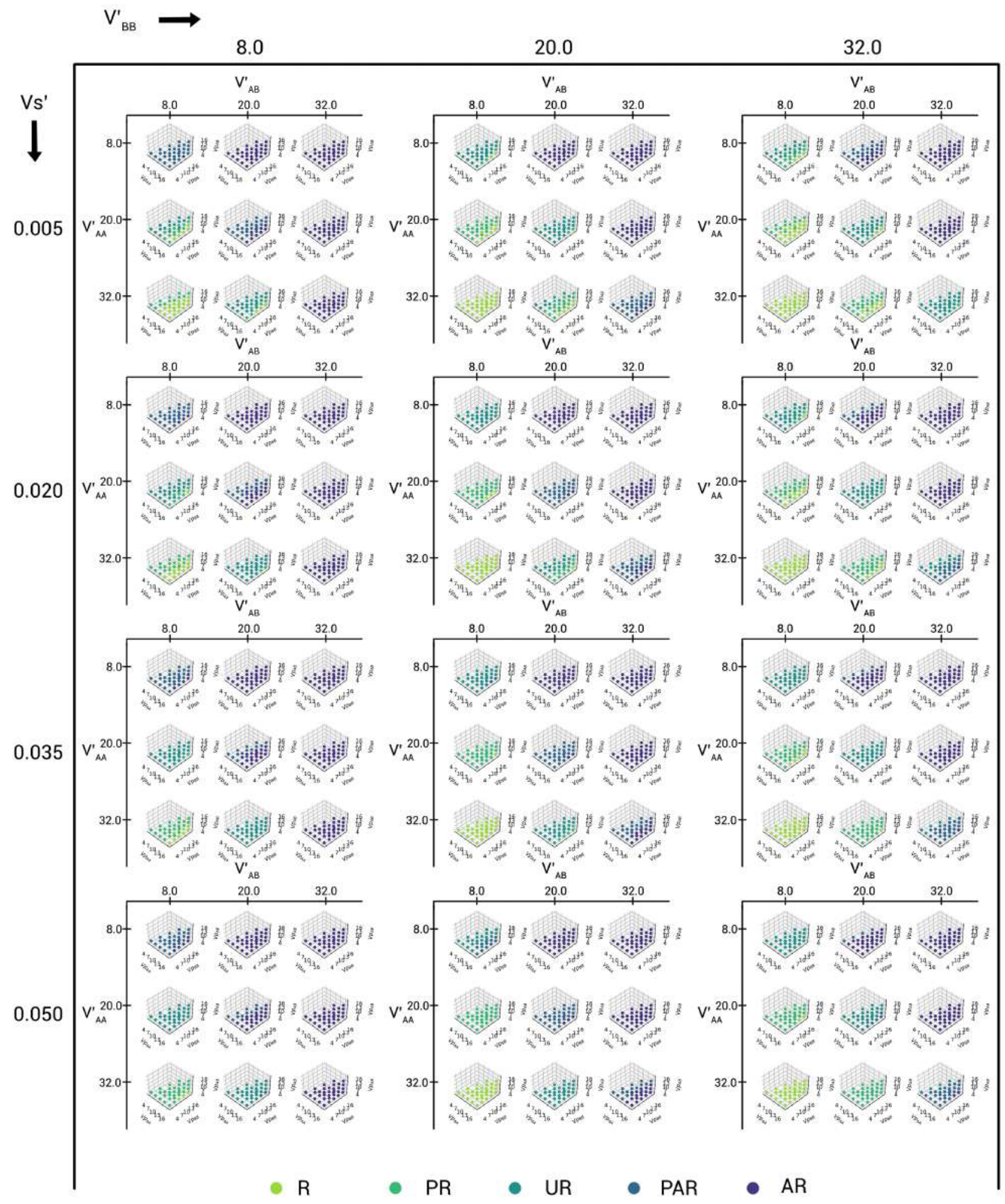

FIG. 11: The plot of domain registration behaviour for all of the parameter space points for the 1:3 DPPC-D34 system, assigned using the KL Divergence analysis. 
either as the Monte-Carlo simulation progressed. We ran extended MC simulations of $10^{9}$ moves to ensure that this wasn't due to a convergence issue, and also saw that these domains persisted throughout the extended simulation. Fig 12 shows a configuration near the end of the extended simulation for the point $0-0-0-2-2-1-4-0-0$ in the 1:3 DPPC-D34 system. Some additional points studied are shown in fig:S9, fig:S10, and fig:S11 in sec.V1] of the S.I. Also, we have shared a movie file (MovieS1.mp4) of a system that lies in the parameter space exhibiting persistent nanodomains as a part of the S.I. The full set of points that we found are collectively described in the supplementary material.

We also plotted a domain size distribution averaged over the last 20 output configurations to more clearly see the sizes of these domains. Fig 13 shows the domain size distribution plot, where we can see that the observed domains seem to range in size from around 80 - 380 lipids without being very elongated, as we confirm from visual observation.

Using typical area per lipid values for DPPC, we can roughly estimate the sizes of these domains, and they fall within the official definition of size of nanodomains of $4-200 \mathrm{~nm}[50]$. With a rough value of area per lipid for DPPC to be $64 \AA^{2}$, these domains fall roughly within $7-16 \mathrm{~nm}$ in size.

The interesting aspect of this observation is that our Hamiltonian only incorporates simple enthalpic and entropic contributions, which suggests that the interplay of the entropic contribution to push towards smaller aggregations as well as the interleaflet enthalpic interaction between registered/anti-registered domains might be sufficient to preserve and evolve small domains. It must be noted that the points where we observe this tend to have stronger interleaflet self interaction, as well as stronger interaction between DPPCs within a leaflet, with weaker D34-D34 and DPPC-D34 interactions. The interaction parameters within the leaflets seem quite justified, and the relative interleaflet enthalpic parameters could form a basis for further investigation of this phenomenon.

\section{DISCUSSION}

From the trends and patterns observed above from the parameter space plots, we find that the interleaflet entropic interaction term promotes anti-registration in the DPPC-D23 and DPPC-D34 systems that we have studied. We also found that anti-registration was strongly promoted in the systems with the 1:3 and 3:1 DPPC-DX ratio in each leaflet, with domain registration seen only for points with very high values of $V_{A A}^{\prime}$ (DPPC-DPPC interleaflet enthalpic interaction strength parameter). In all the 6 systems, we also see that the increase in domain registration due to increase in $V_{A A}^{\prime}$ is greater than that due to increase in $V_{B B}^{\prime}$ (corresponding to DX-DX interleaflet enthalpic interaction) in all 6 systems. It is therefore clear that the saturated lipid tail termini in the interleaflet region provide the primary enthalpic driving force for domain registration, whereas the unsaturated lipid tail termini provide an entropic counter that promotes domain anti-registration. This is supported by studies showing that lipid acyl chain dynamics is a key contributor to interleaflet coupling [51]. The enthalpic contribution of the unsaturated lipids is also important, however, in increasing the coupling between leaflets. We see from the increased fraction of $\mathrm{R}$ and $\mathrm{AR}$ points in the DPPC-D34 systems that the higher D34-D34 interleaflet enthalpic interaction compared to that of D23-D23 allows the system to more easily converge to registered or antiregistered states. This is more difficult in the DPPC-D23 systems, as a weaker coupling between leaflets increases fluctuations in the interleaflet region, thereby showing a more spread out categorization of registration states. This illustrates that the function of the lower position of unsaturation is, therefore, to simply improve coupling between the leaflets both through enthalpic and entropic contributions, and that the system converges to a registered or anti-registered state depending on the relative enthalpic and entropic contributions, which can vary significantly based on the specific lipids in the system.

We think that the behaviour demonstrated in the paper by S. Zhang and X. Lin [4 can be explained by our interpretation of stronger interleaflet coupling in the DPPC-D34 system as compared to the DPPC-D23 system due to a lower position of unsaturation in the D34 lipids instead of their original explanation that suggested that the D23 lipids had interleaflet interactions closer to that of the saturated lipid DPPC, leading to lesser enthalpic driving force for registration. We predict that the number and size of packing defects in the interleaflet region would be higher in a system where the unsaturated lipid has a higher position of unsaturation, and that there will be better packing in a system where the unsaturated lipid has a lower position of unsaturation [52, 53]. Since the increase in interleaflet entropic interaction strength $\left(V_{S}^{\prime}\right)$ can also be loosely interpreted as a rise in temperature the way our Hamiltonian is defined, our observation that lower values of $V_{S}^{\prime}$ allow greater number of domain registered points in parameter space also demonstrates the better enthalpic coupling seen in systems at lower temperatures.

We also find that phase-separation and antiregistration are strongly correlated when $V_{A B}^{\prime}$ is high, whereas phase separation and registration are correlated when $V_{A A}^{\prime}$ and $V_{B B}^{\prime}$ are high and $V_{A B}^{\prime}$ is low. This indicates that the registration and anti-registration tendencies of a domain can influence the phase-separation in the other leaflet. This has also been observed in multiple theoretical studies earlier [23], [24] and further supports the feasibility of our model. Additionally, we observe the presence of thermodynamically stable nanodomains with our curvature-independent Hamiltonian. These observations are in line with the direct, probe-free imaging of model and bioderived membrane using cryogenic electron microscope [54 and fluorescence resonance en- 

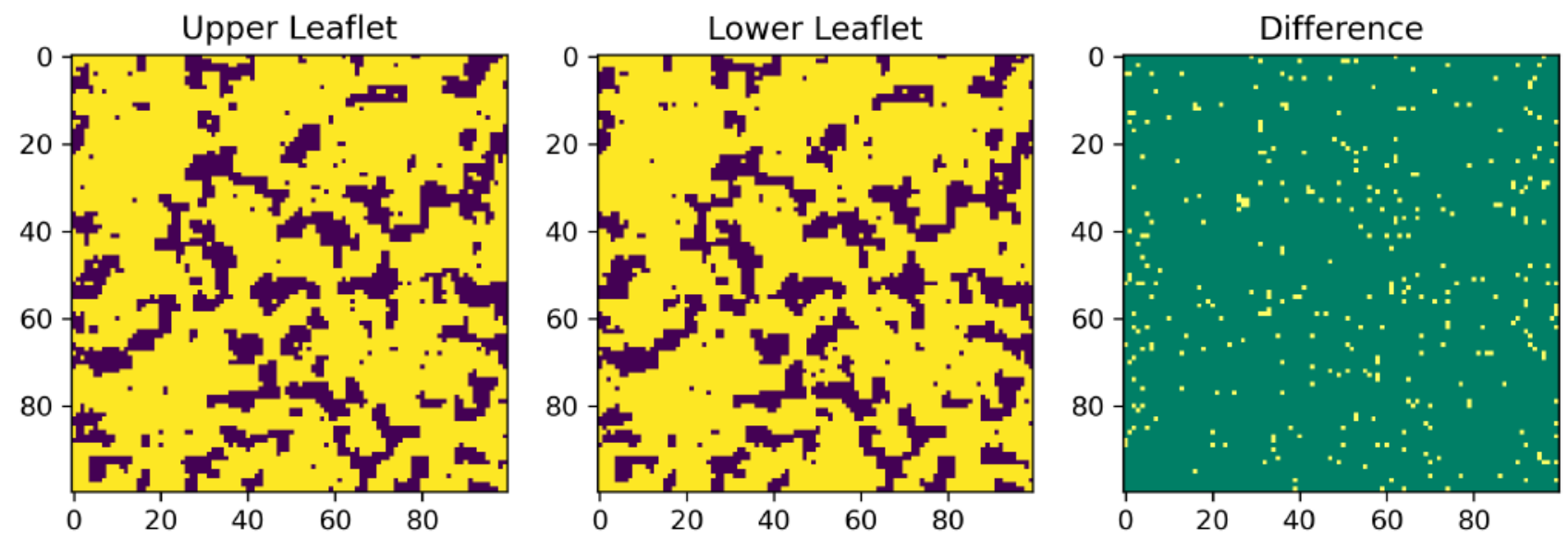

FIG. 12: Configuration near the end of the $10^{9}$ step extended simulation showing the observed nanodomains for the 1:3 DPPC-D34 system at parameter space point 0-0-0-2-2-1-4-0-0. The colours in the plot have the same meaning as in fig.5.

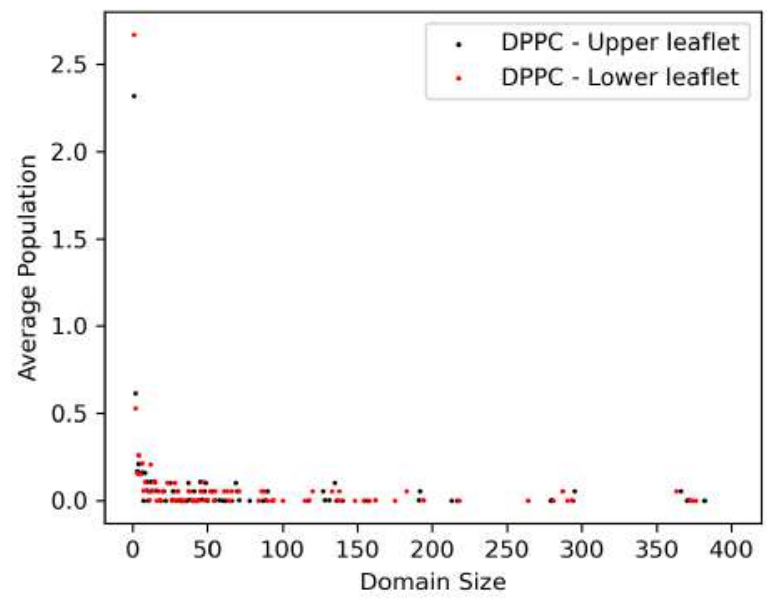

FIG. 13: Domain size distribution plot for DPPC in the upper leaflet (black dots) and lower leaflet (red dots). A large fraction of the DPPC population lies in domains of sizes ranging from $80-380$

ergy transfer (FRET) and, electron spin resonance (ESR) studies on model quaternary lipid systems [55], which provide evidence towards the existence of nanodomains in cell membranes. The experimental evidences are supported by theoretical studies by DW Allender and M Schick [56] where the authors suggested that inhomogeneity in biological membranes might exist in the form of a microemulsion of Lo and Ld regions. They attribute this to spontaneous curvature of the membrane, bending modulus, surface tension as well as the energy cost of formation of concentration gradients. We believe our observation supports the microemulsion hypothesis and provide a possible direction of further investigation.
This study primarily focused on the position of unsaturation, and not on factors like tail interdigitation that have also been shown to affect interleaflet coupling. We avoided this, as we already had too many parameters in the Hamiltonian to work with. However, for the lipids that we worked with, it shouldn't be very significant as the lengths of the lipids were roughly similar. The DPPC-D23 and DPPC-D34 systems are very good model systems to isolate the effect of the difference in position of unsaturation due to their similarity in all other aspects.

\section{CONCLUSIONS}

In this study, we have demonstrated that the domain registration and anti-registration depends on strength of coupling between the leaflets, which is modulated by the position of unsaturation in the tail of the unsaturated lipids. The lower the position of unsaturation, the better the coupling. Whether the system equilibrates to a registered or anti-registered state or something in between depends on the relative enthalpic and entropic contribution to this interleaflet coupling from the interactions of lipid tail termini in the interleaflet region. The lipids with lower positions of unsaturation likely improve coupling due to them having a larger number of configurations where their tail termini are in the interleaflet region and not bent away.

\section{SUPPORTING MATERIAL}

1. supporting_information.pdf : supporting information 
2. movieS1.avi : Persistent registered nanodomain movie

\section{AUTHOR CONTRIBUTIONS}

Anand Srivastava conceived the idea and designed the experiments in consultation with Akshara Sharma. Aniruddha Seal and Sahithya Iyer developed the protocol for creating the artificial lipid bilayer systems under considerations and set up and ran the all-atom simulations. Anand Srivastava and Akshara Sharma formulated the Hamiltonian functions and Akshara Sharma carried out the Monte Carlo simulation and all the analyses. Akshara Sharma and Anand Srivastava wrote the paper with help of other authors.

\section{ACKNOWLEDGMENTS}

Anand Srivastava (A.S.) would like to thank Prof. Madan Rao for discussions. Financial support from the Indian Institute of Science-Bangalore and the highperformance computing facility "Beagle" setup from grants by a partnership between the Department of Biotechnology of India and the Indian Institute of Science (IISc-DBT partnership programme) are greatly acknowledged. A.S. thanks the startup grant provided by the Ministry of Human Resource Development of India and the early career grant from the Department of Science and Technology of India. A.S. also thanks the DST for the National Supercomputing Mission grant. FIST program sponsored by the Department of Science and Technology and UGC, Centre for Advanced Studies and Ministry of Human Resource Development, India is gratefully acknowledged by the authors.
[1] G. G. Putzel, M. J. Uline, I. Szleifer, and M. Schick, Interleaflet coupling and domain registry in phase-separated lipid bilayers, Biophysical journal 100, 996 (2011).

[2] J. J. Williamson and P. D. Olmsted, Registered and antiregistered phase separation of mixed amphiphilic bilayers, Biophysical journal 108, 1963 (2015).

[3] T. R. Galimzyanov, P. I. Kuzmin, P. Pohl, and S. A. Akimov, Undulations drive domain registration from the two membrane leaflets, Biophysical journal 112, 339 (2017).

[4] S. Zhang and X. Lin, Lipid acyl chain cis double bond position modulates membrane domain registration/anti-registration, Journal of the American Chemical Society 141, 15884 (2019), pMID: 31532653, https://doi.org/10.1021/jacs.9b06977

[5] E. J. Shimshick and H. M. Mcconnell, Lateral phase separation in phospholipid membranes, Biochemistry 12, 2351 (1973).

[6] R. F. De Almeida, A. Fedorov, and M. Prieto, Sphingomyelin/phosphatidylcholine/cholesterol phase diagram: boundaries and composition of lipid rafts, Biophysical journal 85, 2406 (2003).

[7] C. Dietrich, L. Bagatolli, Z. Volovyk, N. Thompson, M. Levi, K. Jacobson, and E. Gratton, Lipid rafts reconstituted in model membranes, Biophysical journal 80, 1417 (2001).

[8] S. L. Veatch and S. L. Keller, Organization in lipid membranes containing cholesterol, Physical review letters $\mathbf{8 9}$, 268101 (2002).

[9] S. L. Veatch and S. L. Keller, Separation of liquid phases in giant vesicles of ternary mixtures of phospholipids and cholesterol, Biophysical journal 85, 3074 (2003).

[10] S. L. Veatch and S. L. Keller, Seeing spots: complex phase behavior in simple membranes, Biochimica et Biophysica Acta (BBA)-Molecular Cell Research 1746, 172 (2005).

[11] J. Korlach, P. Schwille, W. W. Webb, and G. W. Feigenson, Characterization of lipid bilayer phases by confocal microscopy and fluorescence correlation spectroscopy, Proceedings of the National Academy of Sciences 96, 8461 (1999).
[12] C. Schmidt, Y. Barenholz, C. Huang, and T. Thompson, Monolayer coupling in sphingomyelin bilayer systems, Nature 271, 775 (1978).

[13] K. Simons and E. Ikonen, Functional rafts in cell membranes, nature 387, 569 (1997).

[14] K. Simons and D. Toomre, Lipid rafts and signal transduction, Nature reviews Molecular cell biology 1, 31 (2000).

[15] H. M. McConnell and M. Vrljic, Liquid-liquid immiscibility in membranes, Annual review of biophysics and biomolecular structure 32, 469 (2003).

[16] P. Sharma, R. Varma, R. Sarasij, K. Gousset, G. Krishnamoorthy, M. Rao, S. Mayor, et al., Nanoscale organization of multiple gpi-anchored proteins in living cell membranes, Cell 116, 577 (2004).

[17] Y. U. Katagiri, N. Kiyokawa, and J. Fujimoto, A role for lipid rafts in immune cell signaling, Microbiology and immunology 45, 1 (2001).

[18] F. Magnani, C. G. Tate, S. Wynne, C. Williams, and J. Haase, Partitioning of the serotonin transporter into lipid microdomains modulates transport of serotonin, Journal of Biological Chemistry 279, 38770 (2004).

[19] L. K. Cuddy, W. Winick-Ng, and R. J. Rylett, Regulation of the high-affinity choline transporter activity and trafficking by its association with cholesterol-rich lipid rafts, Journal of neurochemistry 128, 725 (2014).

[20] M. B. Stone, S. A. Shelby, M. F. Núñez, K. Wisser, and S. L. Veatch, Protein sorting by lipid phase-like domains supports emergent signaling function in b lymphocyte plasma membranes, Elife 6, e19891 (2017).

[21] D. Lingwood and K. Simons, Lipid rafts as a membraneorganizing principle, science 327, 46 (2010).

[22] J. Bodosa, S. S. Iyer, and A. Srivastava, Preferential protein partitioning in biological membrane with coexisting liquid ordered and liquid disordered phase behavior: underlying design principles, The Journal of Membrane Biology , 1 (2020).

[23] D. W. Allender and M. Schick, Phase separation in bilayer lipid membranes: effects on the inner leaf due to coupling to the outer leaf, Biophysical journal 91, 2928 
(2006).

[24] G. G. Putzel and M. Schick, Phase behavior of a model bilayer membrane with coupled leaves, Biophysical journal 94, 869 (2008).

[25] M. Schick, Strongly correlated rafts in both leaves of an asymmetric bilayer, The Journal of Physical Chemistry B 122, 3251 (2017).

[26] A. Horner, S. A. Akimov, and P. Pohl, Long and short lipid molecules experience the same interleaflet drag in lipid bilayers, Physical review letters 110, 268101 (2013).

[27] T. R. Galimzyanov, R. J. Molotkovsky, M. E. Bozdaganyan, F. S. Cohen, P. Pohl, and S. A. Akimov, Elastic membrane deformations govern interleaflet coupling of lipid-ordered domains, Physical review letters 115, 088101 (2015).

[28] J. J. Williamson and P. D. Olmsted, Kinetics of symmetry and asymmetry in a phase-separating bilayer membrane, Physical Review E 92, 052721 (2015).

[29] P. W. Fowler, J. J. Williamson, M. S. Sansom, and P. D. Olmsted, Roles of interleaflet coupling and hydrophobic mismatch in lipid membrane phase-separation kinetics, Journal of the American Chemical Society 138, 11633 (2016).

[30] J. D. Perlmutter and J. N. Sachs, Interleaflet interaction and asymmetry in phase separated lipid bilayers: Molecular dynamics simulations, Journal of the American Chemical Society 133, 6563 (2011).

[31] A. Polley, S. Mayor, and M. Rao, Bilayer registry in a multicomponent asymmetric membrane: Dependence on lipid composition and chain length, The Journal of Chemical Physics 141, 064903 (2014)

[32] S. J. Marrink, H. J. Risselada, S. Yefimov, D. P. Tieleman, and A. H. De Vries, The martini force field: coarse grained model for biomolecular simulations, The journal of physical chemistry B 111, 7812 (2007).

[33] S. Jo, T. Kim, V. G. Iyer, and W. Im, Charmm-gui: a web-based graphical user interface for charmm, Journal of computational chemistry 29, 1859 (2008).

[34] S. Kim, J. Lee, S. Jo, C. L. Brooks III, H. S. Lee, and W. Im, Charmm-gui ligand reader and modeler for charmm force field generation of small molecules (2017).

[35] C. J. Knight and J. S. Hub, Memgen: A general web server for the setup of lipid membrane simulation systems, Bioinformatics 31, 2897 (2015).

[36] D. Van Der Spoel, E. Lindahl, B. Hess, G. Groenhof, A. E. Mark, and H. J. Berendsen, Gromacs: fast, flexible, and free, Journal of computational chemistry 26, 1701 (2005).

[37] M. J. Abraham, T. Murtola, R. Schulz, S. Páll, J. C. Smith, B. Hess, and E. Lindahl, Gromacs: High performance molecular simulations through multi-level parallelism from laptops to supercomputers, SoftwareX 1, 19 (2015).

[38] T. Darden, D. York, and L. Pedersen, Particle mesh ewald: An $n \log (\mathrm{n})$ method for ewald sums in large systems, The Journal of chemical physics 98, 10089 (1993).

[39] U. Essmann, L. Perera, M. L. Berkowitz, T. Darden, H. Lee, and L. G. Pedersen, A smooth particle mesh ewald method, The Journal of chemical physics 103, 8577 (1995).

[40] S. Nosé and M. Klein, Constant pressure molecular dynamics for molecular systems, Molecular Physics 50, 1055 (1983).
[41] M. Parrinello and A. Rahman, Polymorphic transitions in single crystals: A new molecular dynamics method, Journal of Applied Physics 52, 7182 (1981)

[42] B. Hess, H. Bekker, H. J. Berendsen, and J. G. Fraaije, Lincs: a linear constraint solver for molecular simulations, Journal of computational chemistry 18, 1463 (1997).

[43] N. Metropolis, A. W. Rosenbluth, M. N. Rosenbluth, A. H. Teller, and E. Teller, Equation of state calculations by fast computing machines, The Journal of Chemical Physics 21, 1087 (1953).

[44] P. F. Almeida, Thermodynamics of lipid interactions in complex bilayers, Biochimica et Biophysica Acta (BBA)Biomembranes 1788, 72 (2009).

[45] S. S. Iyer, M. Tripathy, and A. Srivastava, Fluid phase coexistence in biological membrane: Insights from local nonaffine deformation of lipids, Biophysical journal 115, 117 (2018).

[46] S. S. Iyer and A. Srivastava, Degeneracy in molecular scale organization of biological membranes, Soft Matter 16, $6752(2020)$

[47] A. J. Sodt, M. L. Sandar, K. Gawrisch, R. W. Pastor, and E. Lyman, The molecular structure of the liquid-ordered phase of lipid bilayers, Journal of the American Chemical Society 136, 725 (2014).

[48] A. J. Sodt, R. W. Pastor, and E. Lyman, Hexagonal substructure and hydrogen bonding in liquid-ordered phases containing palmitoyl sphingomyelin, Biophysical journal 109, 948 (2015).

[49] S. Kullback and R. A. Leibler, On information and sufficiency, The annals of mathematical statistics 22, 79 (1951).

[50] M. Cebecauer, M. Amaro, P. Jurkiewicz, M. J. Sarmento, R. Šachl, L. Cwiklik, and M. Hof, Membrane lipid nanodomains, Chemical reviews 118, 11259 (2018).

[51] J. Tian, J. Nickels, J. Katsaras, and X. Cheng, Behavior of bilayer leaflets in asymmetric model membranes: atomistic simulation studies, The Journal of Physical Chemistry B 120, 8438 (2016).

[52] M. Tripathy, S. S. Iyer, and A. Srivastava, Molecular origin of spatiotemporal heterogeneity in biomembranes with coexisting liquid phases: Insights from topological rearrangements and lipid packing defects, in Advances in Biomembranes and Lipid Self-Assembly, Vol. 28 (Elsevier, 2018) pp. 87-114.

[53] M. Tripathy, S. Thangamani, and A. Srivastava, Threedimensional packing defects in lipid membrane as a function of membrane order, Journal of Chemical Theory and Computation 16, 7800 (2020)

[54] F. A. Heberle, M. Doktorova, H. L. Scott, A. D. Skinkle, M. N. Waxham, and I. Levental, Direct label-free imaging of nanodomains in biomimetic and biological membranes by cryogenic electron microscopy, Proceedings of the National Academy of Sciences 117, 19943 (2020).

[55] W.-C. Tsai and G. W. Feigenson, Lowering line tension with high cholesterol content induces a transition from macroscopic to nanoscopic phase domains in model biomembranes, Biochimica et Biophysica Acta (BBA)Biomembranes 1861, 478 (2019).

[56] D. W. Allender and M. Schick, Recent experiments support a microemulsion origin of plasma membrane domains: Dependence of domain size on physical parameters, Membranes 10, 167 (2020). 
[57] M. Parrinello and A. Rahman, Polymorphic transitions in single crystals: A new molecular dynamics method, Journal of Applied physics 52, 7182 (1981).

[58] R. J. Gowers, M. Linke, J. Barnoud, T. J. E. Reddy, M. N. Melo, S. L. Seyler, J. Domanski, D. L. Dotson, S. Buchoux, I. M. Kenney, et al., MDAnalysis: a Python package for the rapid analysis of molecular dynamics simulations, Tech. Rep. (Los Alamos National Lab.(LANL), Los Alamos, NM (United States), 2019).

[59] N. Michaud-Agrawal, E. J. Denning, T. B. Woolf, and O. Beckstein, Mdanalysis: a toolkit for the analysis of molecular dynamics simulations, Journal of computa- tional chemistry 32, 2319 (2011).

[60] M. C. Blosser, A. R. Honerkamp-Smith, T. Han, M. Haataja, and S. L. Keller, Transbilayer colocalization of lipid domains explained via measurement of strong coupling parameters, Biophysical Journal 109, $2317(2015)$

[61] S. S. Iyer, A. Negi, and A. Srivastava, Interpretation of phase boundary fluctuation spectra in biological membranes with nanoscale organization, Journal of Chemical Theory and Computation 16, 2736 (2020). 
bioRxiv preprint doi: https://doi.org/10.1101/2021.09.29.462263; this version posted September 30, 2021. The copyright holder for this preprint (which was not certified by peer review) is the author/funder, who has granted bioRxiv a license to display the preprint in perpetuity. It is made available under aCC-BY 4.0 International license.

\title{
Enthalpic and Entropic Contributions to Interleaflet Coupling Drive Domain Registration and Anti-registration in Biological Membrane Supplementary Information
}

\author{
Akshara Sharmat \\ Department of Physics, Indian Institute of Science-Bangalore, \\ C. V. Raman Road, Bangalore, Karnataka 560012, India \\ Aniruddha Seal \\ School of Chemical Sciences, \\ National Institute of Science Education and Research, \\ Bhubaneswar, Khurda, Odisha 752050, India \\ Sahithya S. Iyer and Anand Srivastavd \\ Molecular Biophysics Unit, Indian Institute of Science-Bangalore, \\ C. V. Raman Road, Bangalore, Karnataka 560012, India
}

(Dated: September 29, 2021)

\section{CONTENTS}

I. Schematics of the workflow

1

II. Tips and Troubleshooting for the AA System Generation

III. Convergence of MC Simulations

2

IV. Phase Separation plots

2

V. Domain Registration Plots

6

V1. Additional points in parameter space displaying stable nanodomains

References

\section{SCHEMATICS OF THE WORKFLOW}

Fig.1 shows the overall workflow followed in the project. Starting from the hypothesis, we first carried out the all-atom simulations with the appropriate lipid structures for the D23 and D34 lipids to supply the sitevariable to the lattice bilayer Hamiltonian that was developed in parallel. Following this, the parametric study was conducted using this input while varying the other parameters of the Hamiltonian to observe the qualitative trends shown with the variation of each parameter.

* Also at Molecular Biophysics Unit, Indian Institute of ScienceBangalore.

$\dagger$ Currently at Department of Chemistry, University of Illinois Chicago, 845 W. Taylor St., 4500 SES (MC 111), Chicago, IL 60607, U.S.A

$\ddagger$ anand@iisc.ac.in
Finally, we draw conclusions from these trends and interpret the analysed data which is compared to existing results to validate our model.

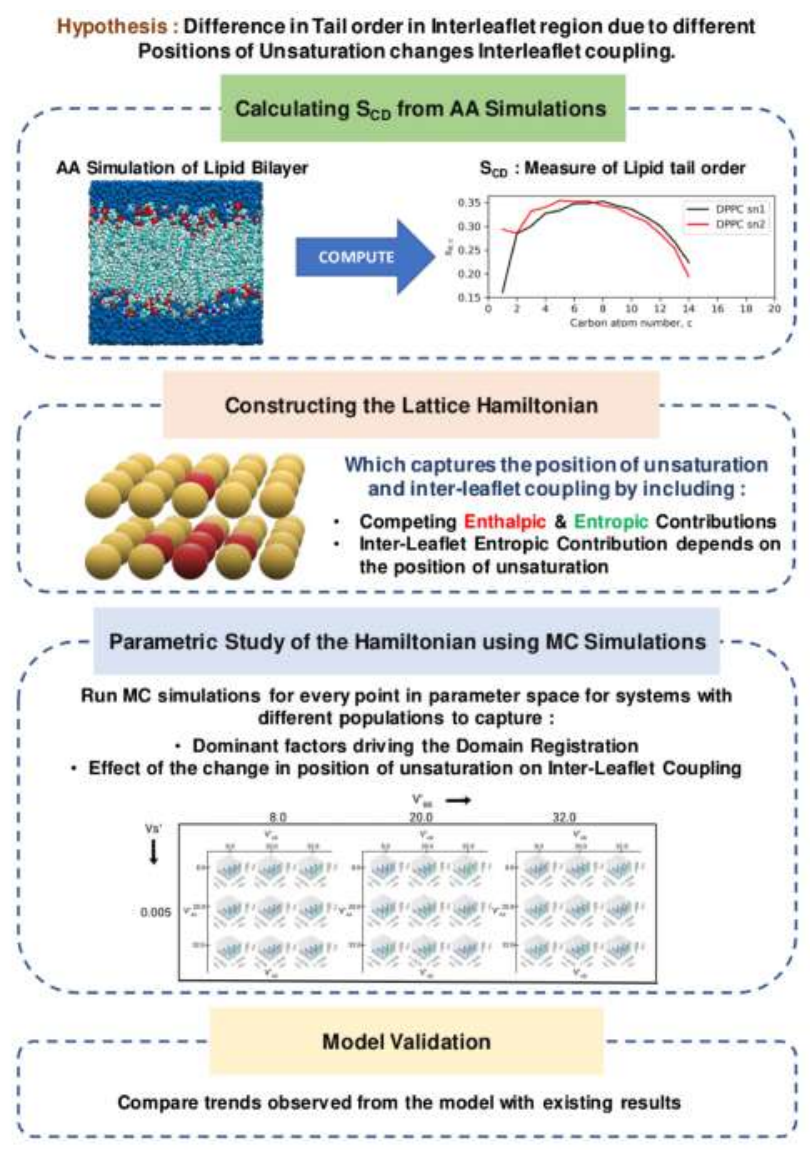

FIG. 1: A schematic showing the workflow adopted in this project. 


\section{TIPS AND TROUBLESHOOTING FOR THE AA SYSTEM GENERATION}

This section contains some extra steps that might be required, as learnt from our experience during the development of the procedure.

- To make asymmetric bilayers, one may make two bilayers using this tool, each of different compositions as required, and then fuse together a leaflet of each.

- The method can require some troubleshooting in the case of appearance of artificial voids. To avoid this, we must visualize the structure obtained and if we see artificial voids in the structure, we must prepare a smaller patch of the system and then replicate it along $\mathrm{X}$ and $\mathrm{Y}$ axis to form the final patch. If the voids still persist, the leaflets need to be moved close to each other, until they overlap.

- The final membrane bilayer structure formed after patching usually shows bad contacts among the waters in the system. Hence, we remove these water molecules, after which we re-solvate the bilayer and remove the water molecules which have penetrated below the headgroups 1, [2] and proceed to the next steps with this new structure. One must also make sure to update the topology file after this.

- A short NPT equilibration (5 ns worked for us) might be required to be performed on the energyminimized structure because the structure of the lipids obtained after the energy minimization is not in their native state. So, running an NVT Equilibration on this system directly can make the system blow up and separate the leaflets on either side of the simulation box.

\section{CONVERGENCE OF MC SIMULATIONS}

In this section, we briefly discuss the convergence of the MC simulations for the various points in parameter space of the six systems studied. Figure 2 shows the energy plots with respect to number of MC moves of four arbitrarily chosen parameter space points from each system. We can see that the systems have reached a stable energy with an acceptably small variance in energy values. The variation of the energy for all points is within $1 \%$ of the final total system energy at that point in parameter space.

Although we haven't plotted the energy vs moves curve for every point in parameter space, we have verified manually that systems with all the different types of final configurations produced during our study indeed show acceptable variation in energy near the end of their simulations.

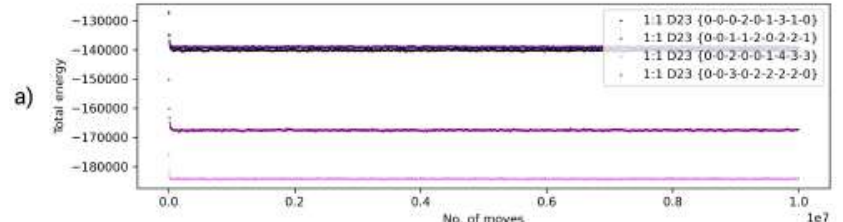

b)

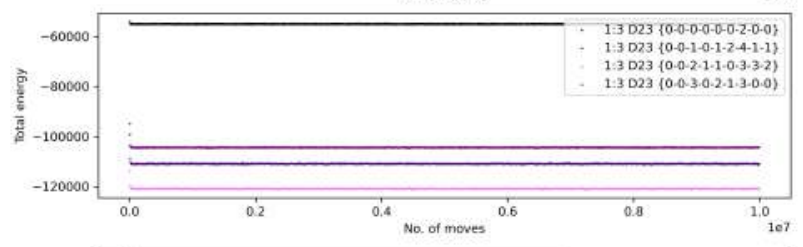

c)
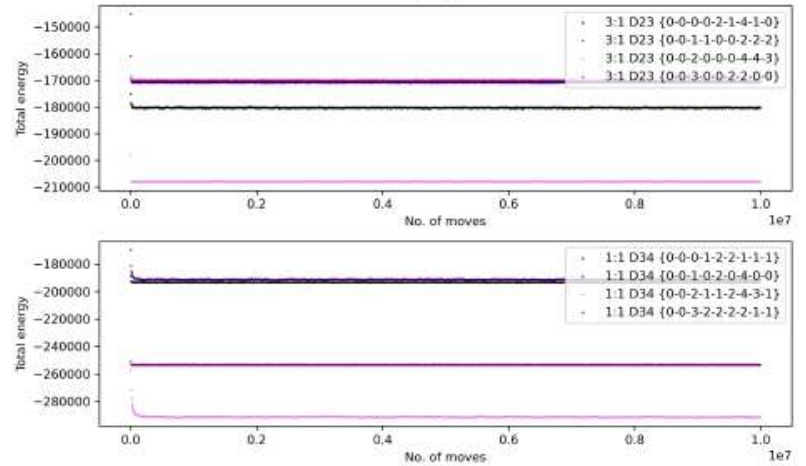

e)

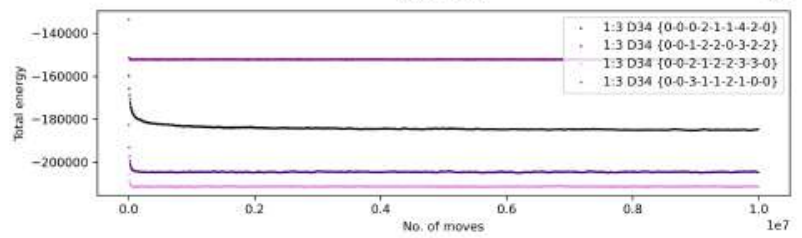

f)

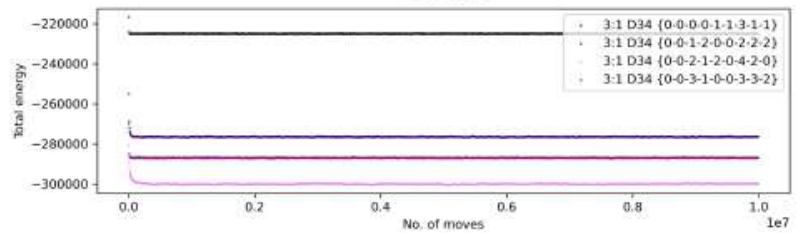

FIG. 2: Total system energy vs. number of MC moves plotted for 4 points from each of the 6 systems, a) 1:1 DPPC-D23 systems, b) 1:3 DPPC-D23 systems, c) 3:1 DPPC-D23 systems, d) 1:1 DPPC-D34 systems, e) 1:3 DPPC-D34 systems, f) 3:1 DPPC-D34 systems. As can be seen, the energy converges quickly and remains steady.

\section{PHASE SEPARATION PLOTS}

This section shows the phase-separtion plots of the systems excluded from the main manuscript, namely the 1:3 DPPC-D23 system in fig 3 , the 3:1 DPPC-D23 system in fig, 4, and the 3:1 DPPC-D34 system in fig 5 . 
bioRxiv preprint doi: https://doi.org/10.1101/2021.09.29.462263; this version posted September 30, 2021. The copyright holder for this preprint (which was not certified by peer review) is the author/funder, who has granted bioRxiv a license to display the preprint in perpetuity. It is made available under aCC-BY 4.0 International license.

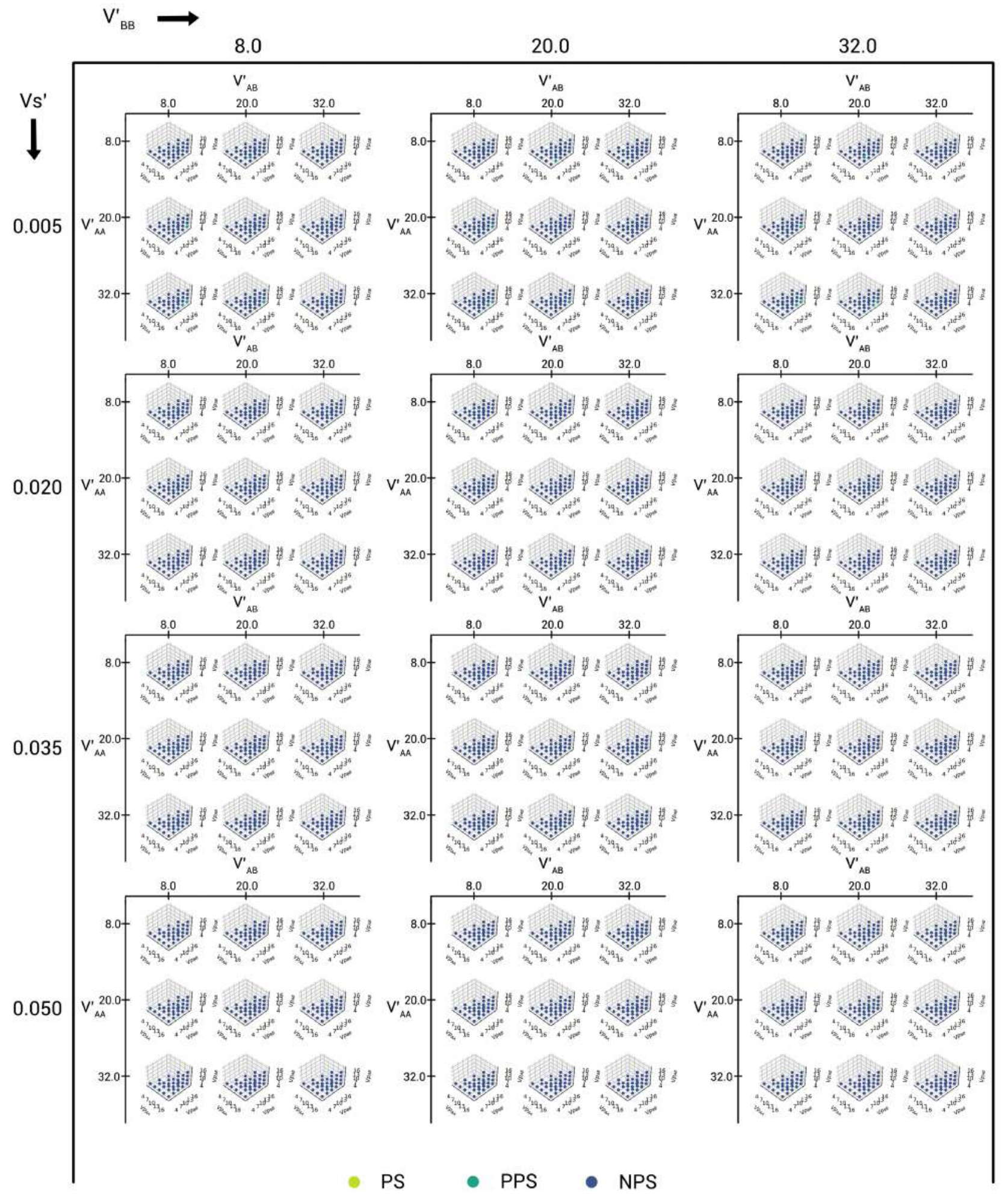

FIG. 3: The plot of phase separation classification for all of the parameter space points for the 1:3 DPPC-D23 system, assigned using the domain size distribution analysis. 
bioRxiv preprint doi: https://doi.org/10.1101/2021.09.29.462263; this version posted September 30, 2021. The copyright holder for this preprint (which was not certified by peer review) is the author/funder, who has granted bioRxiv a license to display the preprint in perpetuity. It is made available under aCC-BY 4.0 International license.

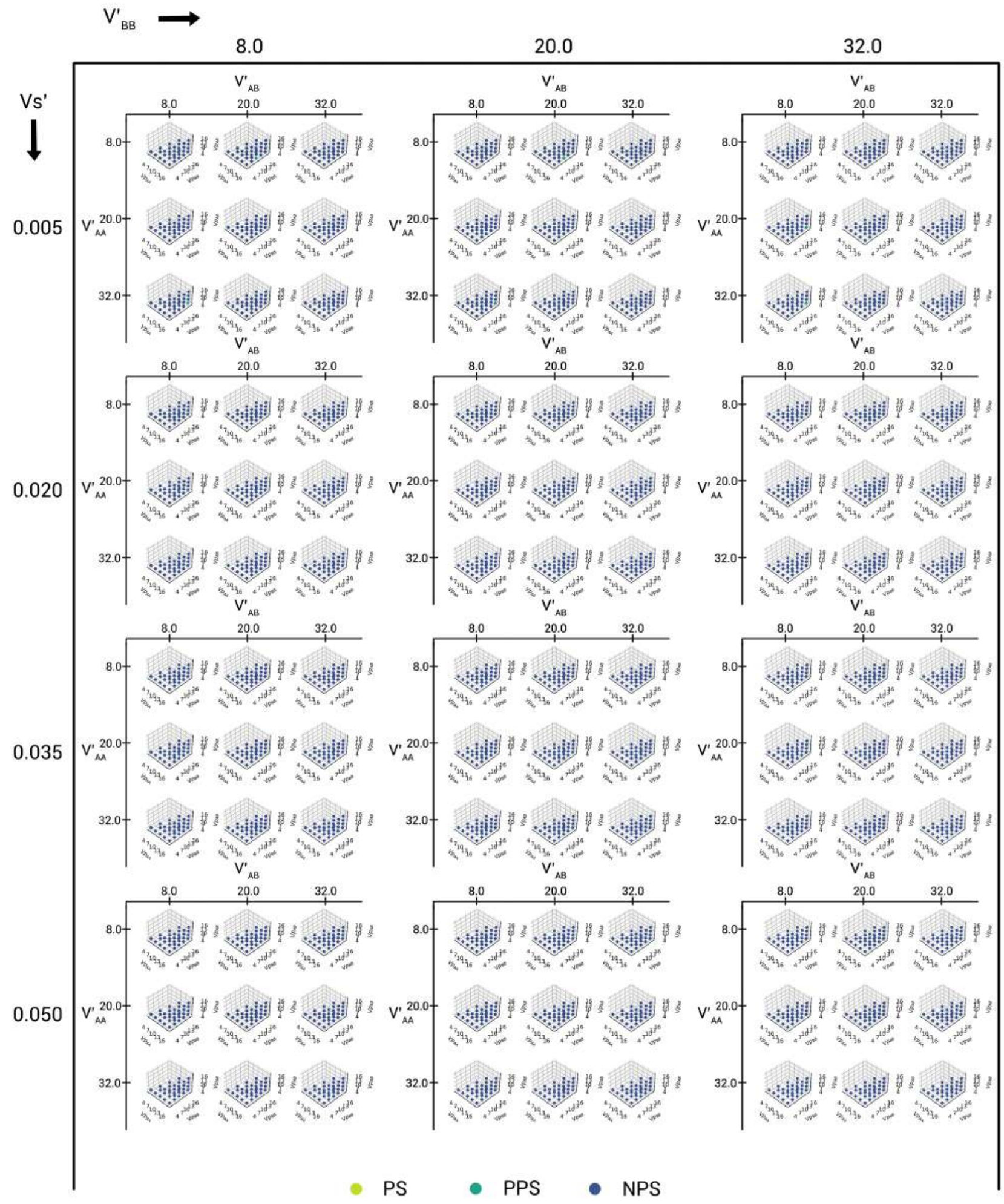

FIG. 4: The plot of phase separation classification for all of the parameter space points for the 3:1 DPPC-D23 system, assigned using the domain size distribution analysis. 
bioRxiv preprint doi: https://doi.org/10.1101/2021.09.29.462263; this version posted September 30, 2021. The copyright holder for this preprint (which was not certified by peer review) is the author/funder, who has granted bioRxiv a license to display the preprint in perpetuity. It is made available under aCC-BY 4.0 International license.

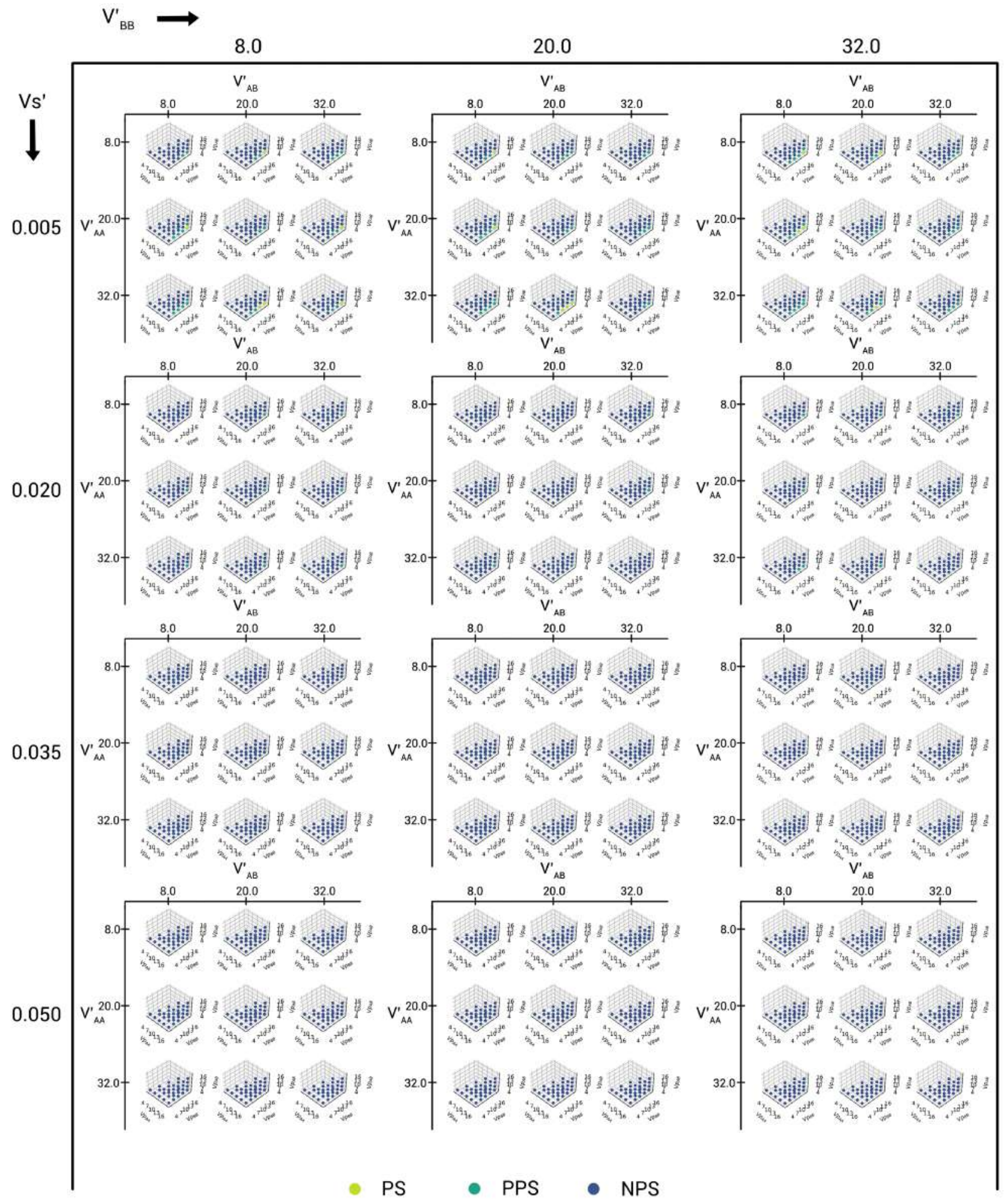

FIG. 5: The plot of phase separation classification for all of the parameter space points for the 3:1 DPPC-D34 system, assigned using the domain size distribution analysis. 
bioRxiv preprint doi: https://doi.org/10.1101/2021.09.29.462263; this version posted September 30, 2021. The copyright holder for this preprint (which was not certified by peer review) is the author/funder, who has granted bioRxiv a license to display the preprint in perpetuity. It is made available under aCC-BY 4.0 International license.

\section{DOMAIN REGISTRATION PLOTS}

This section shows the domain registration plots of the systems excluded from the main manuscript, namely the 1:3 DPPC-D23 system in fig 6, the 3:1 DPPC-D23 system in fig,7, and the 3:1 DPPC-D34 system in fig,8,
VI. ADDITIONAL POINTS IN PARAMETER SPACE DISPLAYING STABLE NANODOMAINS

Some additional points showing formation of stable nanodomains are shown in fig 9 fig 10, and fig 11 The blue coloured squares represent DPPC and the green squares represent D34 in the following images. In the differences map, yellow squares show mismatched/antiregistered sites and green squares represent registered sites. 
bioRxiv preprint doi: https://doi.org/10.1101/2021.09.29.462263; this version posted September 30, 2021. The copyright holder for this preprint (which was not certified by peer review) is the author/funder, who has granted bioRxiv a license to display the preprint in perpetuity. It is made available under aCC-BY 4.0 International license.

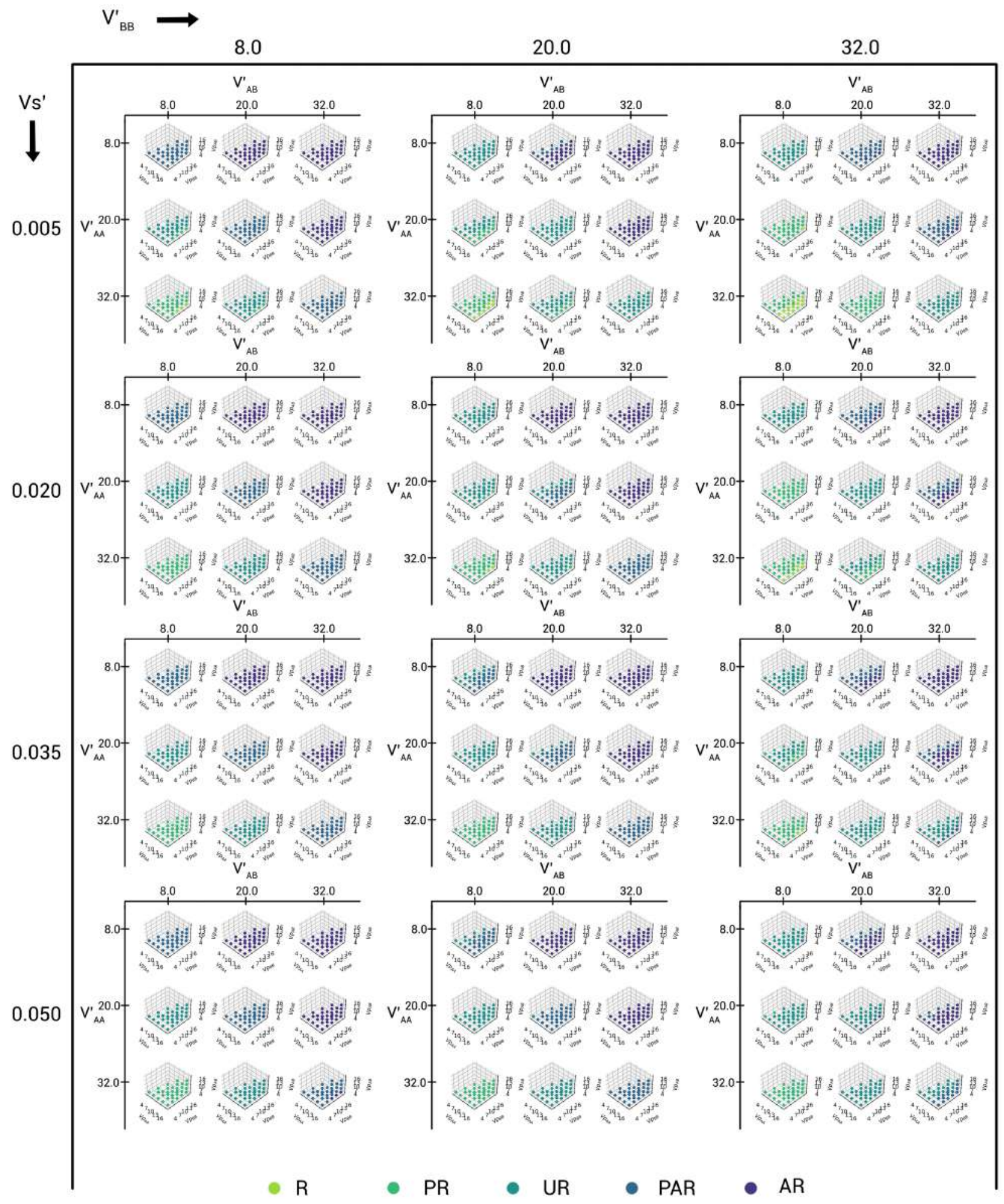

FIG. 6: The plot of domain registration behaviour for all of the parameter space points for the 1:3 DPPC-D23 system, assigned using the KL Divergence analysis. 
bioRxiv preprint doi: https://doi.org/10.1101/2021.09.29.462263; this version posted September 30, 2021. The copyright holder for this preprint (which was not certified by peer review) is the author/funder, who has granted bioRxiv a license to display the preprint in perpetuity. It is made available under aCC-BY 4.0 International license.

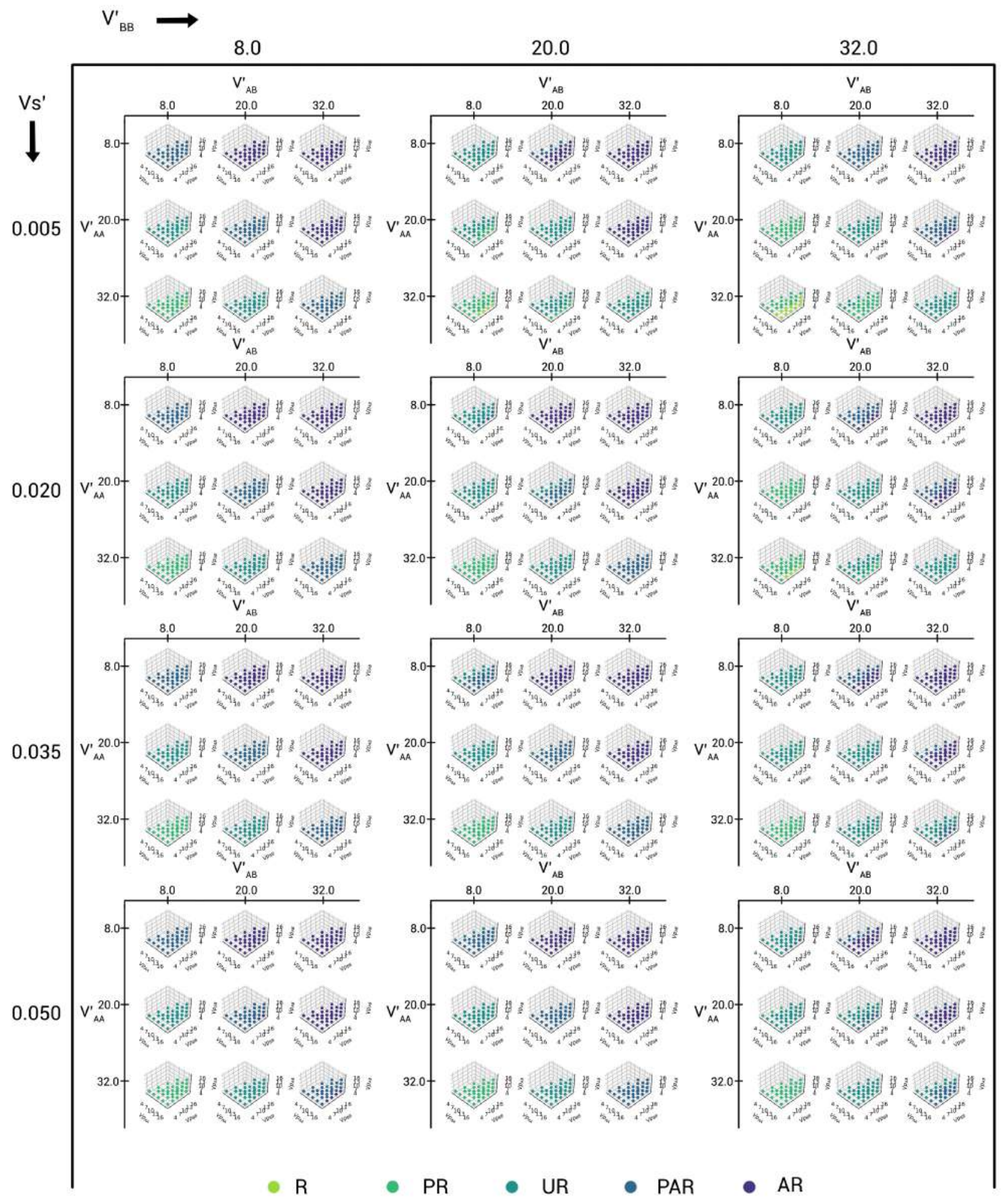

FIG. 7: The plot of domain registration behaviour for all of the parameter space points for the 3:1 DPPC-D23 system, assigned using the KL Divergence analysis. 
bioRxiv preprint doi: https://doi.org/10.1101/2021.09.29.462263; this version posted September 30, 2021. The copyright holder for this preprint (which was not certified by peer review) is the author/funder, who has granted bioRxiv a license to display the preprint in perpetuity. It is made available under aCC-BY 4.0 International license.

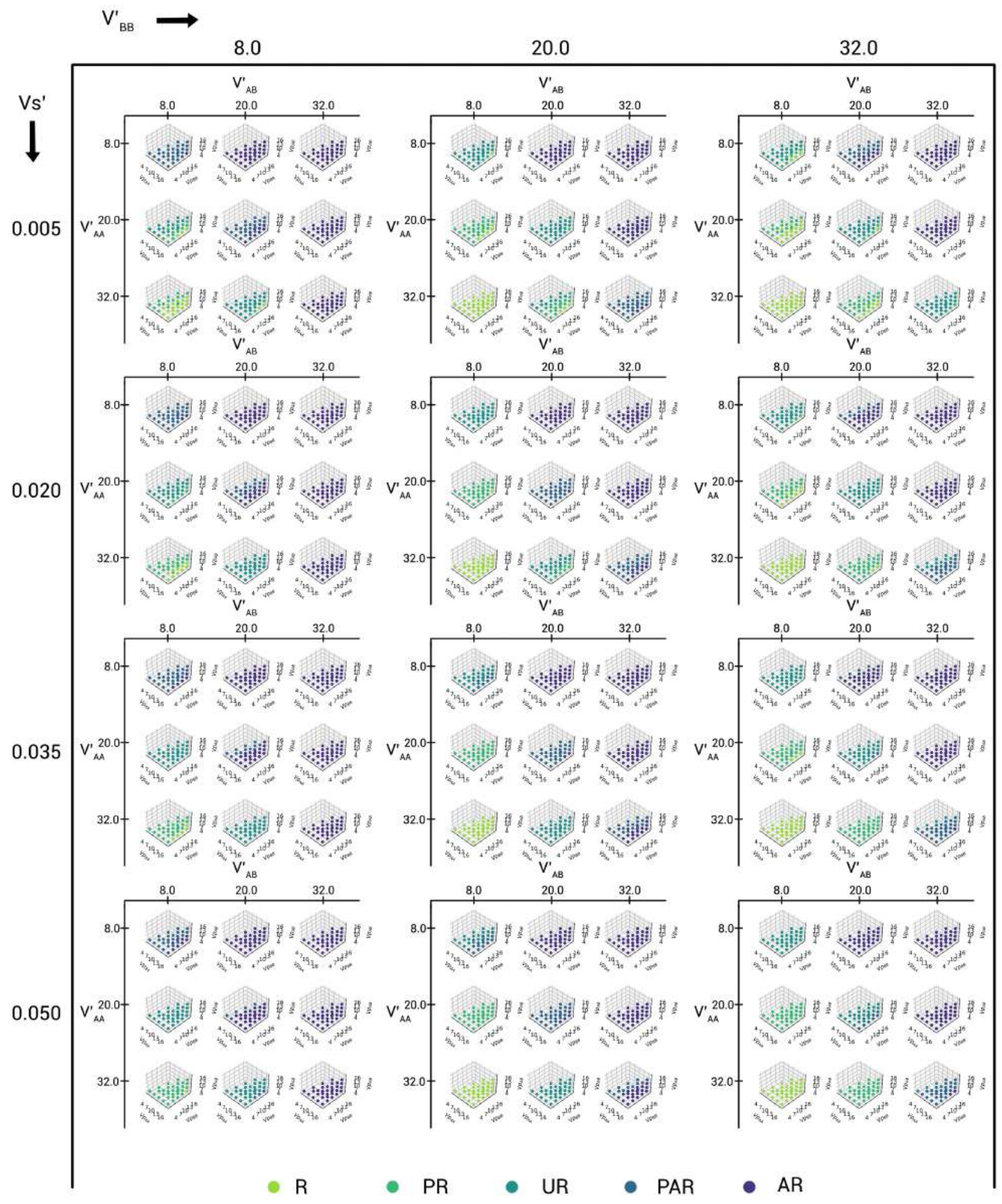

FIG. 8: The plot of domain registration behaviour for all of the parameter space points for the 3:1 DPPC-D34 system, assigned using the KL Divergence analysis. 
bioRxiv preprint doi: https://doi.org/10.1101/2021.09.29.462263; this version posted September 30, 2021. The copyright holder for this preprint (which was not certified by peer review) is the author/funder, who has granted bioRxiv a license to display the preprint in perpetuity. It is made available under aCC-BY 4.0 International license.
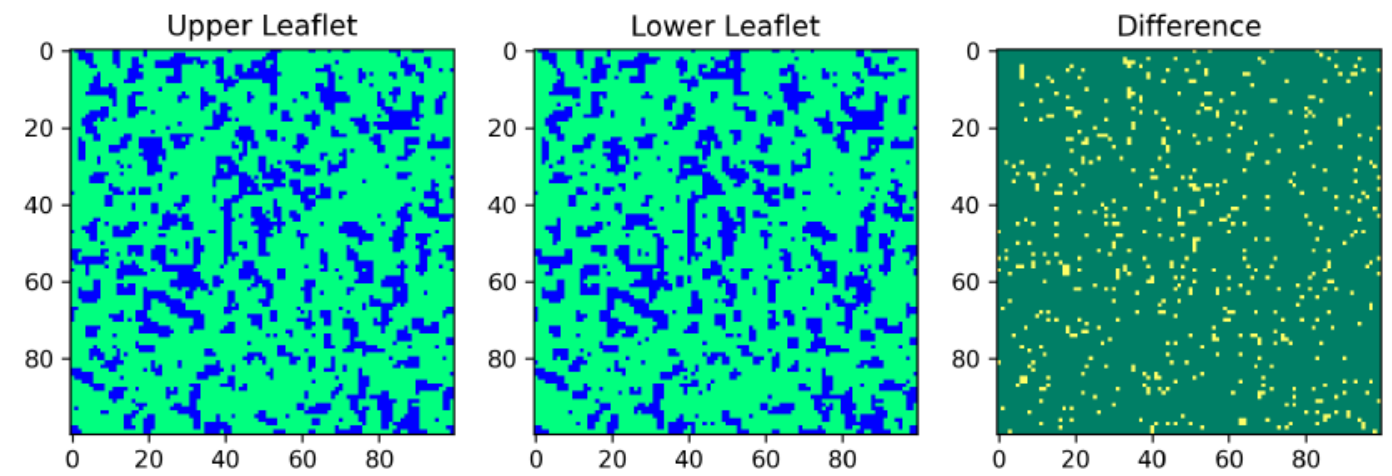

FIG. 9: Configuration near the end of the $10^{9}$ step extended simulation showing the observed nanodomains for the 1:3 DPPC-D34 system at parameter space point 0-0-0-1-1-0-3-1-0.
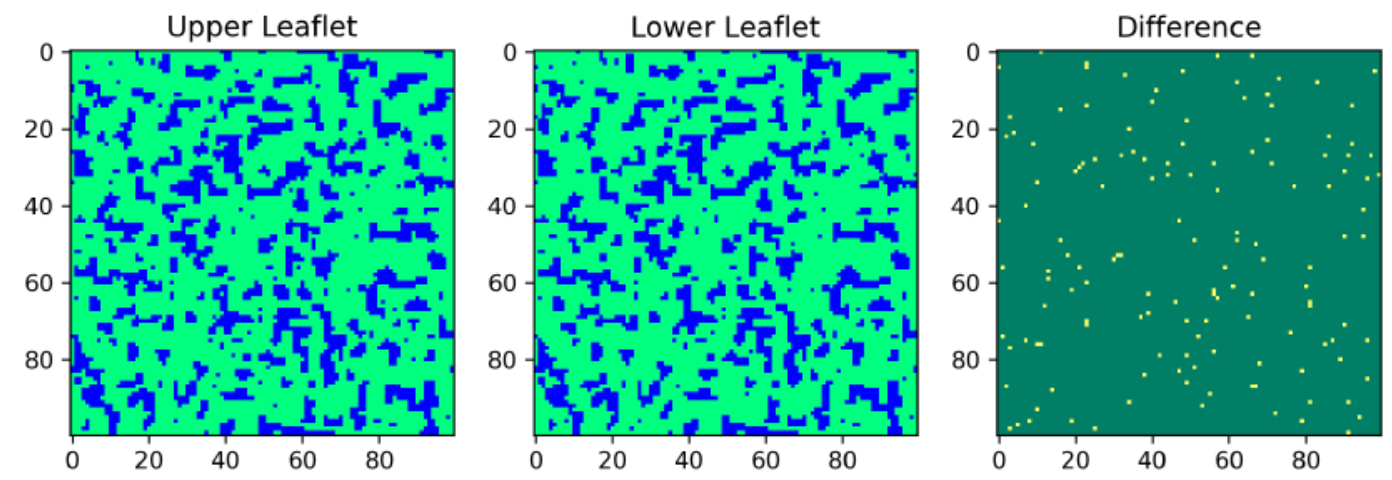

FIG. 10: Configuration near the end of the $10^{9}$ step extended simulation showing the observed nanodomains for the 1:3 DPPC-D34 system at parameter space point 0-0-0-2-1-0-3-1-0.
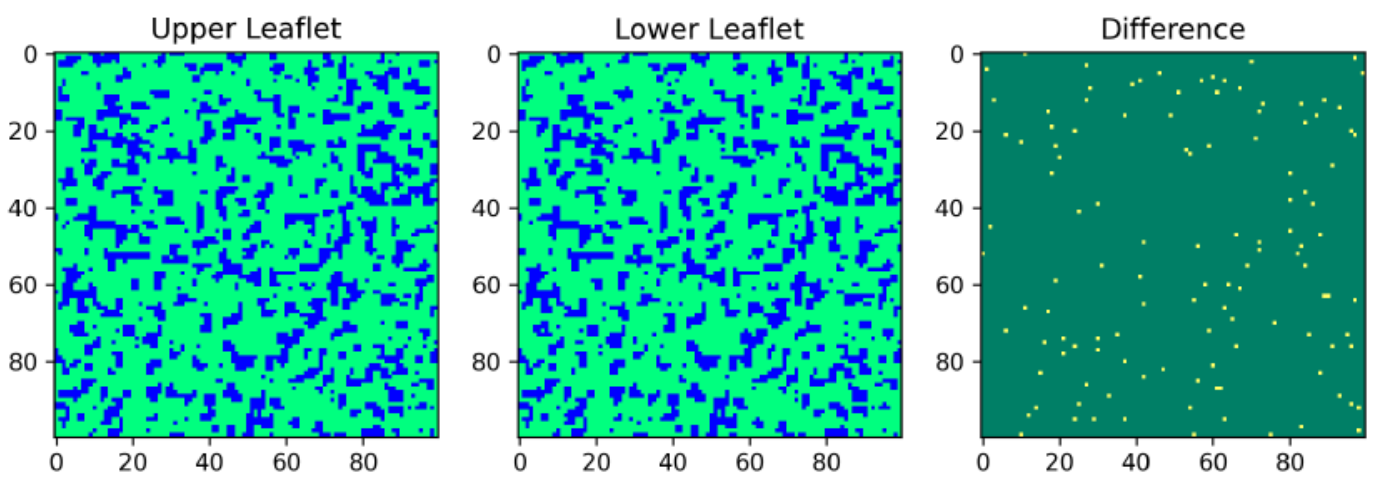

FIG. 11: Configuration near the end of the $10^{9}$ step extended simulation showing the observed nanodomains for the 1:3 DPPC-D34 system at parameter space point 0-0-0-2-2-0-3-0-0.

[1] R. J. Gowers, M. Linke, J. Barnoud, T. J. E. Reddy, M. N. Melo, S. L. Seyler, J. Domanski, D. L. Dotson, S. Buchoux, I. M. Kenney, et al., MDAnalysis: a Python package for the rapid analysis of molecular dynamics simulations, Tech. Rep. (Los Alamos National Lab.(LANL), Los Alamos, NM
(United States), 2019).

[2] N. Michaud-Agrawal, E. J. Denning, T. B. Woolf, and O. Beckstein, Mdanalysis: a toolkit for the analysis of molecular dynamics simulations, Journal of computational chemistry 32, 2319 (2011). 